\title{
Approccio sistemico applicato a territori siti UNESCO
}

\section{System approach applied to territories site UNESCO}

\author{
Luigi Bistagnino
}

Resumo: Um bem histórico ou um território específico foi lentamente moldado ao longo dos anos por uma ação coletiva que o consolidou, e que hoje podemos apreciar e usufruir. Contudo, essa percepção vem sendo gradualmente modificada pela exploração comercial superficial e individualista, prejudicando o meio ambiente e a população local. Esse foi o motivo pelo qual o projeto apresentado nesse artigo foi desenvolvido. Para tanto, foram selecionados três territórios, localizados ao sul da cidade de Turim - Itália, considerados como Patrimônio Cultural pela UNESCO. O objetivo foi despertar a consciência de que é possível valorizar os bens deixados como herança e, concomitantemente, promover o bem-estar dos habitantes locais inseridos em seu contexto social, cultural, produtivo e econômico. Por meio da aplicação da metodologia do Design Sistêmico, foi possível: i) compreender, de maneira holística, os territórios envolvidos; ii) verificar que grande parte da produção dos alimentos consumidos é advindo do exterior, o que provoca problemas de saúde e prejuízos à economia local; iii) redefinir o sistema territorial de produção de alimentos, satisfazendo as exigências alimentares da população, gerando novos postos de trabalho e fonte de renda. A Abordagem Sistêmica traz uma riqueza de recursos, comportamentos e relações sociais que envolvem aqueles que visitam um lugar desconhecido, incentivando-os a voltar para viver novas experiências, um turismo experiencial que participa do ritmo de vida no território, uma relação cultural enriquecedora, e não um surto turístico superficial.

Palavras-chave: território, abordagem sistêmica, cultura, turismo.

Abstract: A Cultural Patrimony or a specific territory was slowly shaped over the years by a collective action that consolidated it, and which today we can enjoy and enjoy. However, this perception has been gradually modified by superficial and individualistic commercial exploitation, damaging the environment and the local population. This paper seeks to understand this modification. To this end, three territories were selected, located to the south of the city of Turin - Italy, considered Cultural Patrimony by UNESCO. The objective was to raise the awareness that it is possible to value assets left as inheritance and, at the same time, to promote the well-being of the local inhabitants 
inserted in their social, cultural, productive and economic context. Through the application of the Systemic Design methodology, it was possible to: 1) understand, in a holistic way, the territories involved; 2) to verify that much of the production of food consumed is coming from abroad, which causes health problems and damages to the local economy; 3) redefine the territorial system of food production, satisfying the food requirements of the population, generating new jobs, and a source of income. The Systemic Approach brings a wealth of resources, behaviors and social relationships that involve those who visit an unknown place, encouraging them to return to live new experiences, experiential tourism that participates in the rhythm of life in the territory, an enriching cultural relationship, and not a superficial tourist outbreak.

Keywords: territory, systemic approach, culture, tourism.

\section{Perché applicare l'Approccio Sistemico a dei territori siti UNESCO}

L'ottenimento della nomina (World Heritage Center oppure Man and the Biosphere Programme - MaB) di un monumento, un sito o un territorio da parte dell'UNESCO1 comporta dapprima una decisione collettiva di enti pubblici e privati di partecipare al riconoscimento e poi anche un considerevole lavoro di squadra per la preparazione documentale delle qualità artistiche, culturali e ambientali che si intendono mettere in evidenza per ottenere l'agognato marchio. E' un coinvolgimento collettivo molto forte utilizzando anche risorse monetarie per la preparazione del dossier e per la presentazione del medesimo al Congresso dell'UNESCO.

Qualora si ottenga la nomina si celebrerà la medesima con un convegno nel quale con orgoglio s'illustrerà tutto il lavoro fatto in modo che i presenti possano apprezzare lo sforzo culturale e materiale compiuto.

Col passare dei giorni però pian piano l'entusiasmo svanisce e le incombenze quotidiane tornano nuovamente ad avere il sopravvento. Si passa dall'orgoglio del risultato

${ }^{1}$ United Nations Educational Scientific and Cultural Organization. ottenuto al fastidio, e presto anche all'opposizione, per tutte le nuove attenzioni che il riconoscimento comporta.

Un bene storico o un ambito specifico sono stati plasmati lentamente negli anni da un agire collettivo che li ha consolidati nella realtà che oggi possiamo apprezzare e percepire; non possono essere "imbalsamati" come statici esempi da guardare o visitare solo pensandoli al passato. Fanno parte del nostro mondo attuale e con essi dobbiamo confrontarci. Su questo piano nascono infatti i problemi di insofferenza a cui ci si riferiva sopra.

Il tutto ci può apparire più chiaro se guardiamo l'evoluzione che in questi ultimi 50 anni c'è stata, per esempio, nello sviluppo delle città sia grandi sia piccole.

Immaginiamo di arrivare con un'autovettura in un agglomerato urbano. Si esce da un casello autostradale (o magari non si arriva da un'autostrada) e ci s’immette nell'arteria stradale che porta al centro città. Il territorio circostante svanisce popolandosi gradualmente di un insieme molto casuale di parallelepipedi prefabbricati con la facciata principale di preferenza tutta in vetro riflettente, di piccole o grandi industrie, di autosaloni o altre attività per nulla coerenti l'una con l'altra che via via formano una quinta caotica lungo la strada impedendone la visione al di là: dalla percezione di un paesaggio aperto e coerente si è ora circondati da un susseguirsi di insegne e messaggi pubblicitari in cui ciascuno guarda se stesso senza relazionarsi con gli altri attori e tantomeno all'intorno. L'agire auto-riferito genera infatti un insieme casuale, caotico e superficiale.

Ogni tanto la quinta è spezzata da grandi parcheggi che attirano ai vari centri commerciali (di scarpe, elettrodomestici, mobili, vestiti, piastrelle, sanitari, cibo ...) per poi ritornare a chiudersi e via via inframmezzarsi con blocchi anonimi di residenze: si è passati dalla zona commerciale e industriale alle residenze di periferia prive di negozi e servizi (non si possono sicuramente annoverare come veri servizi i supermercati). Poi man mano la città si palesa con le sue case e i suoi sporadici negozi sino ad arrivare al cosiddetto centro urbano nuovo con i suoi edifici più accurati degli uffici, delle residenze, dei negozi e poi finalmente al centro storico. 


\section{se il focus è}

l'economia

\section{società}

cultura

ambiente

produzione

\section{saranno ad essa sottomessi}

E' uno spaesamento continuo anche nel centro storico perché anch'esso è

\section{[...] un centro commerciale all'aperto sempre uguale, o meglio ossessivo, con la continua iterazione degli stessi prodotti e degli stessi negozi progettati per essere riconoscibili come appartenenti ad una catena, ma avulsi dal contesto culturale e territoriale in cui si trovano ${ }^{2}$.}

La percezione del coerente e vitale insieme urbano storico viene gradualmente modificata da questo superficiale ed individualistico sfruttamento commerciale.

Con una cultura che si concretizza in questo modo risulta molto difficile, se non impossibile, mantenere in vita le risorse dei beni architettonici ed ambientali che si possiedono. Per il loro mantenimento, ma anche e soprattutto per la nostra sopravvivenza, è necessario sviluppare un paradigma culturale diverso da quello attuale, ma coerente con il sistema naturale e con la nostra vita biologica.

Per questo motivo si è sviluppato il progetto di ricerca dell'applicazione dell'Approccio Sistemico a dei territori posti a Sud della città di Torino che sono siti UNESCO (World Heritage Center e MaB) con la finalità di far nascere la consapevolezza di poter valorizzare nel presente i beni che ci sono stati lasciati in eredità e che potremo lasciare inseriti in un intorno sociale, culturale, produttivo ed

\footnotetext{
2 Da microMACRO, Edizoni Ambiente, Milano 2014.
}

Schema di come attualmente è impostato l'agire quotidiano sia singolo sia collettivo.

economico che li mantenga vivi e ne valorizzi la vita e il benessere degli abitanti insediati.

\section{Approccio Sistemico ${ }^{3}$}

Le azioni che svolgiamo quotidianamente avallano, senza che ce ne rendiamo conto, un paradigma culturale e comportamentale che la nostra società condivide e rinforza con i comportamenti e le decisioni di tutti a qualunque livello si operi. Ognuno partecipa a consolidare il modello cultuale esistente senza porsi alcuna domanda e senza neanche riflettere se ciò che si fa sia giusto o sbagliato e se le conseguenze delle azioni possano nuocere ad altri direttamente o indirettamente.

Questo modo di agire ha forti conseguenze negative sia sugli altri sia su noi stessi.

La prima e forse più importante considerazione è che siamo abituati a dei comportamenti e a delle scelte che mirano a risolvere solo le necessità individuali vivendo sempre più rinchiusi in una sfera personale auto-riferita. Si perde del tutto la coscienza di far parte di un ambiente comune. Ognuno, sia nel piccolo sia nel grande, detta le proprie regole e cerca di prevaricare il prossimo. Non ci sono più obiettivi comuni cui tendere per cercare di realizzarli in uno sforzo collettivo condiviso: la società si disgrega sempre più ed emergono con virulenza l'accumulo e l'ostentazione di denaro.

\footnotetext{
${ }^{3}$ Vedi L. Bistagnino, microMACRO, Edizoni Ambiente, Milano 2014.
} 


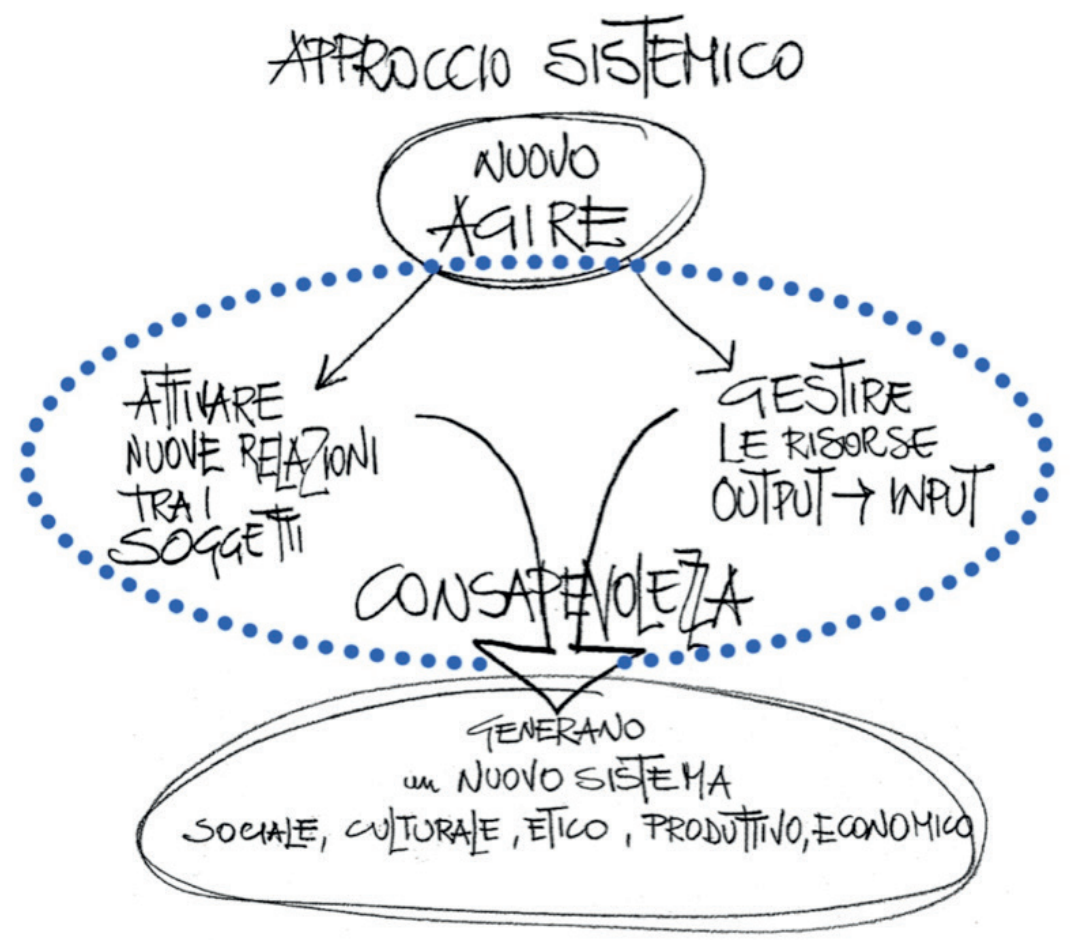

Schema concettuale del nuovo agire sistemico.

La nostra stessa vita viene continuamente misurata sul possesso materiale di qualcosa: "l'avere" è la visualizzazione dei valori che il nostro sistema culturale, economico e sociale mette in primo piano considerando il prodotto come il perno attorno al quale fanno leva tutte le considerazioni.

Mentre sino ad ora si è prestata attenzione alle quantità e qualità delle materie prime e alle loro precipue caratteristiche, per il nostro futuro sarà altrettanto interessante ed essenziale focalizzare l'attenzione non solo su quanto potrà entrare in un sistema ma soprattutto su quanto ne potrà uscire.

Questa visione fa infatti nascere un progetto più allargato e complesso, che abbraccia tutta la filiera produttiva, in cui le problematiche inerenti gli scarti di lavorazione vengono poste sullo stesso livello degli approvvigionamenti e degli utilizzi delle materie prime. Si dovranno principalmente approfondire le qualità degli output e non solo le quantità, perché è proprio da quelle che ne potranno scaturire i reali futuri utilizzi. Vale a dire che si potranno mettere in relazione diverse situazioni produttive in modo che le specifiche qualità degli output di una produzione possano diventare l'input di un'altra.

Se gli output da problema diventano risorsa, con conseguente valore economico, nasce l'interesse nel considerarli parte attiva di un processo e nel valorizzare le loro qualità intrinseche modificando le lavorazioni o i processi che portano a degradarli. L'attenzione si sposta sul mantenere inalterate le proprietà che li rendono appetibili, per non perdere il loro valore economico di scambio, ottenendo al contempo un formidabile risultato: una cultura produttiva tendente a EMISSIONI ZERO.

Si mette in atto un nuovo agire che ha in se la naturalezza di concepire le relazioni come degli utili vincoli collaborativi reciproci che ci legano ad una rete e ad un contesto.

Si passa da una visone quantitativa ad una qualitativa. 
Questa positività, che nasce da nuovi rapporti tra le persone, che come soggetti consapevoli interagiscono l'un l'altro in un reciproco ascolto e collaborazione, unisce le diverse unità trasformandole in un sistema coerente in cui la forza di tutti diventa la forza di ognuno. I singoli legati nell'azione collettiva svolgono una piccola azione che però nell'insieme si trasforma esponenzialmente in un grande risultato. Ognuno dipende dal comportamento dei vicini, ma allo stesso tempo mantiene un grado di autonomia tale da renderlo capace di influenzare positivamente l'intorno e collaborare ai cambiamenti a livello complessivo dell'insieme.

Il comportamento dell'insieme sociale diventa simile a quello di uno stormo di uccelli, di un banco di pesci o di uno sciame di insetti in cui ogni componente agisce nel proprio intorno locale, ma le sue azioni si ripercuotono sulla direzione e sulla forma dell'insieme per adattarsi alle condizioni esterne che via via si presentano.

Le nuove relazioni tra i soggetti si concretizzano nella nuova gestione qualitativa delle risorse in cui gli output di uno sono gli input di un altro in un fluire continuo. Questo scambio reciproco consolida le relazioni tra i vari soggetti unendoli in sistemi produttivi positivi che tendono ad emissioni zero, si sviluppano localmente e generano un'Economia Blu .

Questa nuova consapevolezza ha un'influenza generatrice naturale, mette in atto un agire collettivo che crea un nuovo sistema sociale, culturale, etico, produttivo ed economico e che si diffonde in tutto il territorio locale facendo evolvere il macro sistema lineare esistente in quello sistemico.

Le ricadute generali sono intuibili e toccano veramente tutte le attività esistenti. Dai positivi rapporti sociali che consolideranno le relazioni tra le persone, al rafforzamento della cultura materiale che avrà ricadute sul sistema produttivo, alla valorizzazione del territorio con l'utilizzo corretto delle risorse locali generando salute e benessere, alla forte diminuzione della logistica che si trasformerà in micro spostamenti, all'economia che, tramite i flussi e le relazioni, diventerà una solida e resiliente rete di micro economia locale e che unirà positivamente tutti i soggetti coinvolti generando nuovi posti di lavoro.

La consapevolezza di operare per il bene comune farà spontaneamente parte dell'agire di ognuno.

Non è un mondo onirico, ma quanto succede in Natura in cui ogni singolo è parte vitale dell'insieme complessivo a cui appartiene e che contribuisce a generare.

\section{Il progetto sistemico}

Per facilitare la comprensione della visione sistemica, che è semplice nella sua formulazione ma estremamente difficile nella sua concretizzazione, viene ora descritta l'applicazione pratica dell'Approccio Sistemico nel progetto di ricerca recentemente realizzato su tre territori posti a Sud della città di Torino, che sono anche siti UNESCO.

\section{Il rilievo olistico}

Per agire è necessario conoscere. Non una conoscenza di singoli fatti, di situazioni e di quantificazioni, ma il saper vedere l'insieme delle relazioni che li connettono e in base alle quali si crea l'attuale realtà.

Con questi occhi bisogna quindi avviare l'analisi sulla situazione esistente nei territori considerati, che nel caso specifico sono

- il Pianalto di Poirino,

- la Dorsale dei laghi di cava del Po,

- il Feudo dei 9 Merli.

Su questa base inizia un'attenta riflessione per arrivare a definire, per i singoli territori, le varie positività e negatività riscontrate. L'insieme di queste ultime costituisce il punto di partenza del nuovo progetto: è appunto attraverso la considerazione di vederle come leve per il cambiamento che si può iniziare il percorso di trasformazione dello stato attuale.

4 Vedi G. Pauli, Blue Economy 2.0, Edizioni Ambiente 2015. 
Questa documentazione critica dello stato dell'arte è quello che l'Approccio Sistemico indica appunto come rilievo olistico.

E' l'indicazione della qualità e poi delle quantità che ogni processo coinvolge. Questa indagine approfondisce anche tutte le caratteristiche climatiche, culturali, ambientali, sociali e produttive del territorio; tutte le relazioni che avvengono nel contesto ambientale in cui l'attività è collocata; tutte quelle che si sviluppano al di fuori del territorio locale (nazionale, europeo, extra europeo) ed anche tutte quelle che s'instaurano all'interno della medesima nelle singole azioni che la compongono.

Per una comprensione semplice ed intuitiva che avvalori fortemente la percezione dell'insieme attuale è necessario procedere a piccoli passi con schemi visualizzanti graficamente

- il territorio con le sue caratteristiche climatiche, culturali, ambientali, sociali e produttive.

- le relazioni generate,

- le risorse impiegate in ingresso (input) e generate in uscita (output),

- le diverse azioni di cui si compone un'attività con i relativi input ed output,

\section{Territorio con le sue caratteristiche}

Le analisi indicate con i relativi schemi portano naturalmente a comprendere come il territorio, nel quale avvengono le diverse attività, abbia una rilevanza conoscitiva fondamentale. Risulta quindi utile rappresentare, in un cartellone con immagini e fotografie, le sue caratteristiche paesaggistiche e climatiche in modo da poter entrare percettivamente in relazione con il luogo in cui si opera ed anche approfondire tutte le altre componenti materiali e immateriali che lo contraddistinguono:

- la cultura con le tradizioni, le festività, il folclore, le sagre, gli eventi e l’architettura,

- le risorse ambientali, la flora e la fauna,

- i prodotti tipici e, collegati ad essi, anche i piatti tipici,

- le attività artigianali e industriali,

- il turismo con le attrazioni, stagionali o no, e gli sport che si praticano.

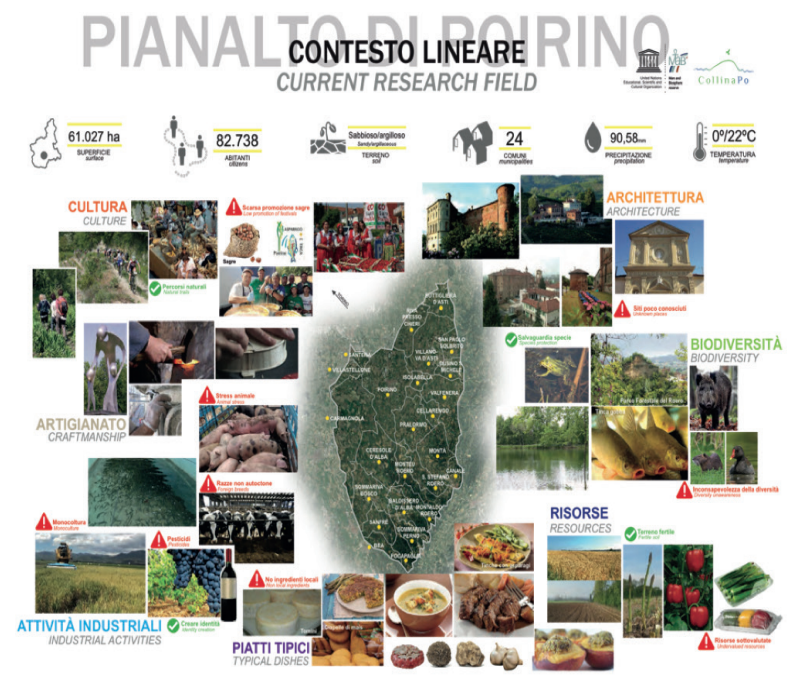

Rappresentazione sintetica del contesto del Pianalto di Poirino con le positività e le negatività riscontrate. 
Lo stretto collegamento tra i vari livelli di approfondimento consente di avere una chiara indicazione di come si svolga realmente la vita del luogo con il coinvolgimento complessivo di tutti gli attori e di poter così fare una riflessione cosciente delle positività, ma anche delle negatività riscontrate.

\section{Relazioni generate}

Chi gestisce un'attività in generale è molto occupato nel coordinare il complesso delle operazioni necessarie allo svolgimento quotidiano e cerca di rendere il più semplice possibile l'approvvigionamento delle risorse necessarie. E' cioè assorbito dall'attività corrente e la sua attenzione non va al di fuori di essa.

Bisogna cercare di ampliare questa visione, guardarsi attorno e vedere quali siano realmente le relazioni intrecciate con l'intorno e con i diversi fornitori di materie e servizi necessari, ma soprattutto capire quale sistema si stia generando. Per far ciò basta semplicemente partire dalla propria attività e collegare con una linea tutte quelle che prima e dopo siano connesse alla propria: prima sino a raggiungere l'origine di provenienza degli input e dopo sino al consumatore finale.

Questa operazione porterà ad allargare utilmente la visione dal proprio contesto a quello di tutti quelli che sono effettivamente coinvolti. E' come realizzare un filmato multiplo che contemporaneamente fa vedere le azioni di tutti gli attori delle varie attività, compresa la propria, che nell'insieme formano il sistema complessivo al quale si appartiene.

Si potranno così capire tutte le positività e negatività che l'attività sta contribuendo a generare e di cui si è corresponsabili.

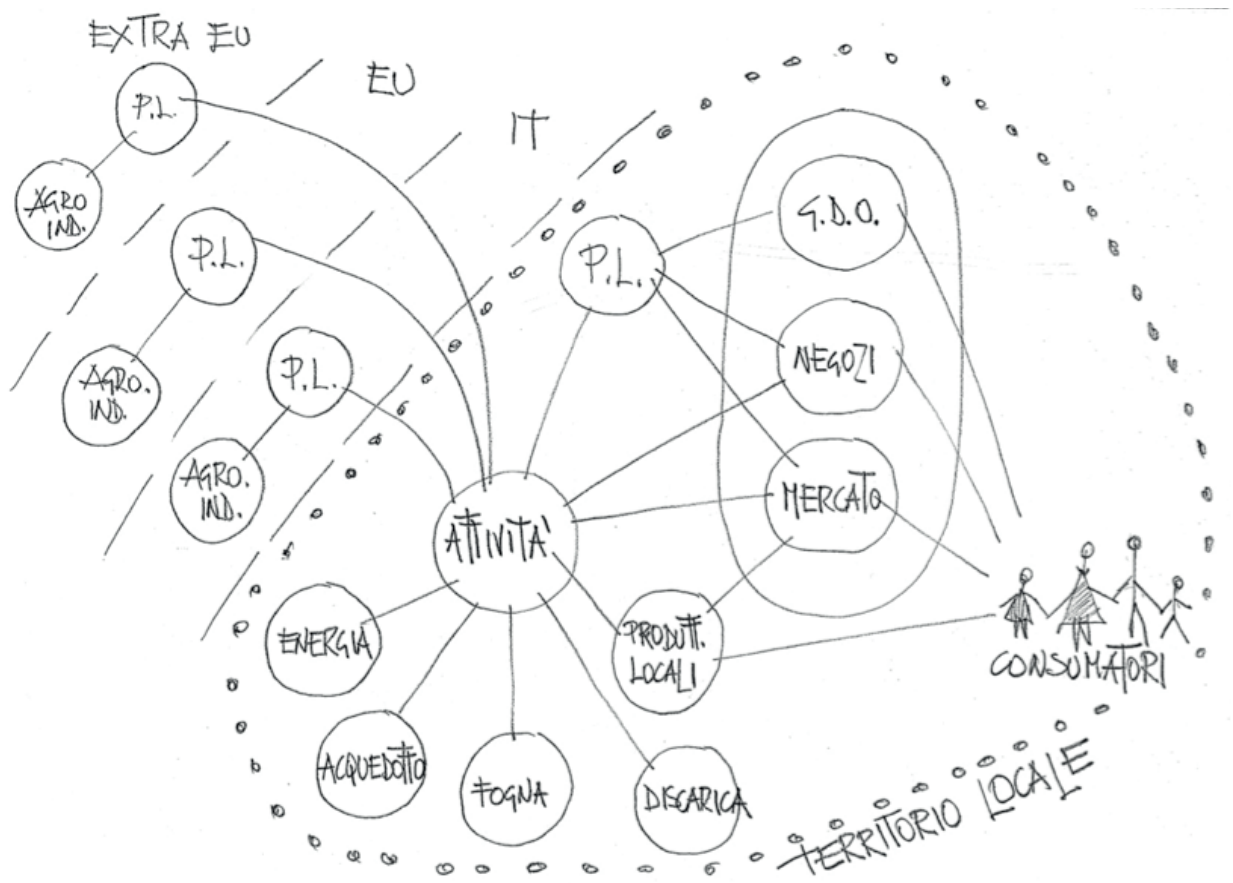

Schema delle relazioni che un'attività genera ed anche il territorio di riferimento dal quale provengono. Le risorse possono provenire da Piattaforme di Logistica (P. L.) collegate a fornitori di Agro Industrie (Agro. Ind.) situati in diversi luoghi Italia (IT) Europa $(E U) O$ anche oltre oceano (Extra EU). I prodotti realizzati possono essere distribuiti alle Grandi Distribuzioni Organizzate dei supermercati (G. D. O.) oppure a negozi o mercati locali (Mercato). Chiaramente vedendo le relazioni si può capire molto bene quali siano i rapporti con il proprio territorio. 


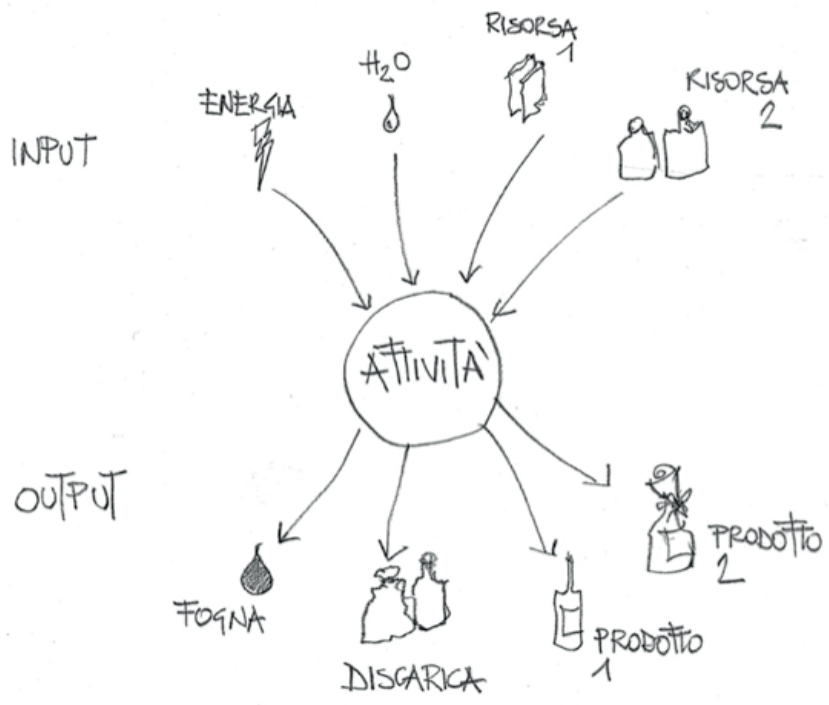

Schema delle risorse che un'attività utilizza in ingresso (input) e produce in uscita (output).

\section{Risorse impiegate in ingresso (input) e generate in uscita (output)}

A questo livello, ponendo al centro la propria attività, si visualizzano tutte le risorse necessarie per lo svolgimento produttivo separando quelle utilizzate in entrata (input) da quelle risultanti in uscita (output). In queste ultime si comprendono sia i prodotti realizzati sia tutte le emissioni e la materia risultanti dal processo produttivo.

L'indicazione da dove provengano gli input e dove vadano gli output fornisce un chiaro riferimento per collegarsi allo schema precedente delle relazioni attivate.

\section{Azioni che compongono un'attività con i relativi input ed output}

Le attività produttive sono costituite da diverse azioni che nella loro sequenza temporale e funzionale costituiscono l'insieme della produzione.

La visualizzazione nella loro successione consente di poter indicare per ciascuna di queste tutti gli input e gli output, che prima sono stati indicati complessivamente. La scomposizione delle azioni consente altresì di individuare quelle fondamentali, quelle che maggiormente utilizzano input o generano output e di collegarli alle attività dalle quali vengono presi o inviati.

Il risultato che si ottiene è una percezione complessiva dell'attività con i vari flussi delle risorse divisi per le singole azioni, ma che ora si possono vedere anche nel loro sviluppo temporale. La variante tempo è fondamentale per poter recuperare, soprattutto nel caso delle attività legate al cibo, la consapevolezza dello svolgersi delle stagioni nell'arco dell'anno e della possibilità di poter avere o meno i prodotti maturi, coerenti con esse, nel territorio in cui ci si trova. 

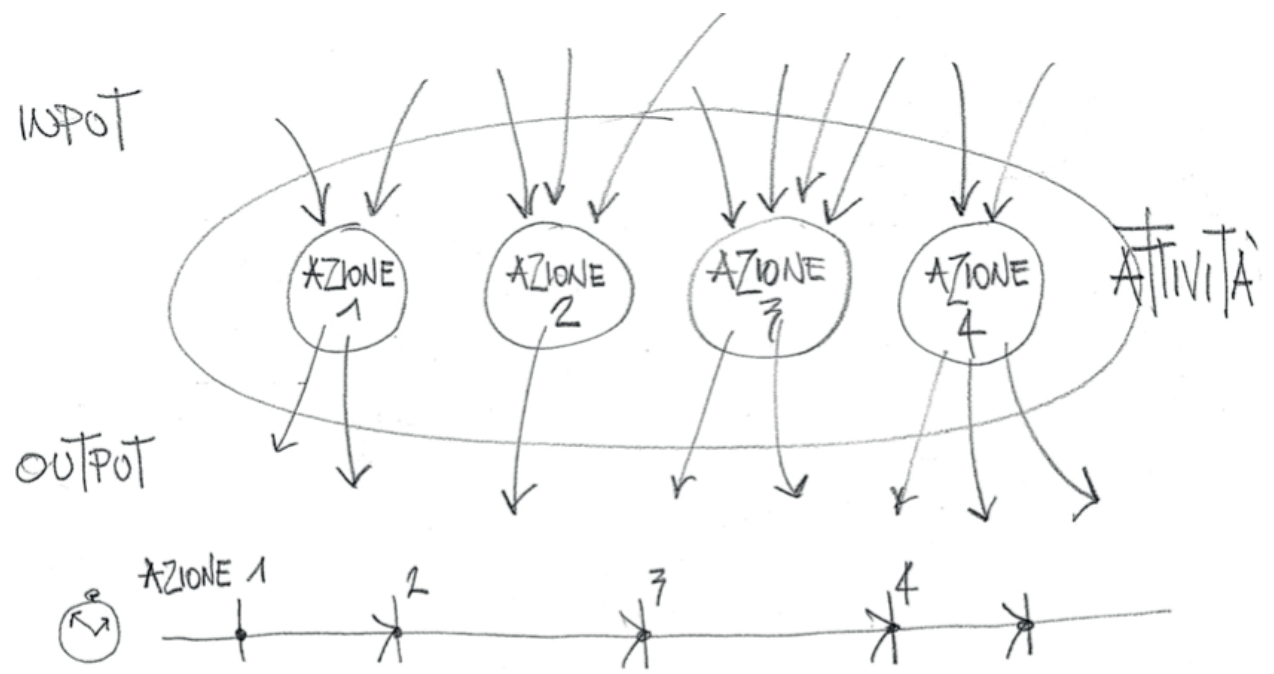

Schema delle azioni che compongono un'attività; il riferimento degli input e degli output alle singole azioni permette di comprenderne le qualità intrinseche ed anche il peso che ciascuna ha rispetto alle altre. La componente tempo, asincrona con lo schema, mette invece in risalto quando le singole azioni vengono effettuate e anche il loro riferimento stagionale.

\section{Situazione attuale}

Il risultato degli approfondimenti, seguendo gli schemi grafici delle relazioni, delle risorse impiegate e delle azioni componenti le singole attività, è sintetizzato nel grafico rappresentante il sistema messo in atto dalle relazioni attuali. Le filiere considerate delle attività primarie (Latte, Carne, Cereali e Frutta \& Verdura) presenti nei territori generano tutte le altre attività sino ad arrivare ai consumatori.

Si crea una rappresentazione su livelli multipli che interagiscono contemporaneamente

- quello locale (formato dall'area agricola e da quella residenziale),

- quello nazionale,

- quello europeo,

- quello extra europeo.

Le attività primarie sono insediate localmente, ma prendono soprattutto le risorse necessarie da altri livelli e principalmente spediscono a questi i propri prodotti; il territorio locale riceve solo una marginalità produttiva che è fintamente locale in quanto generata da flussi provenienti anche da oltre oceano. 


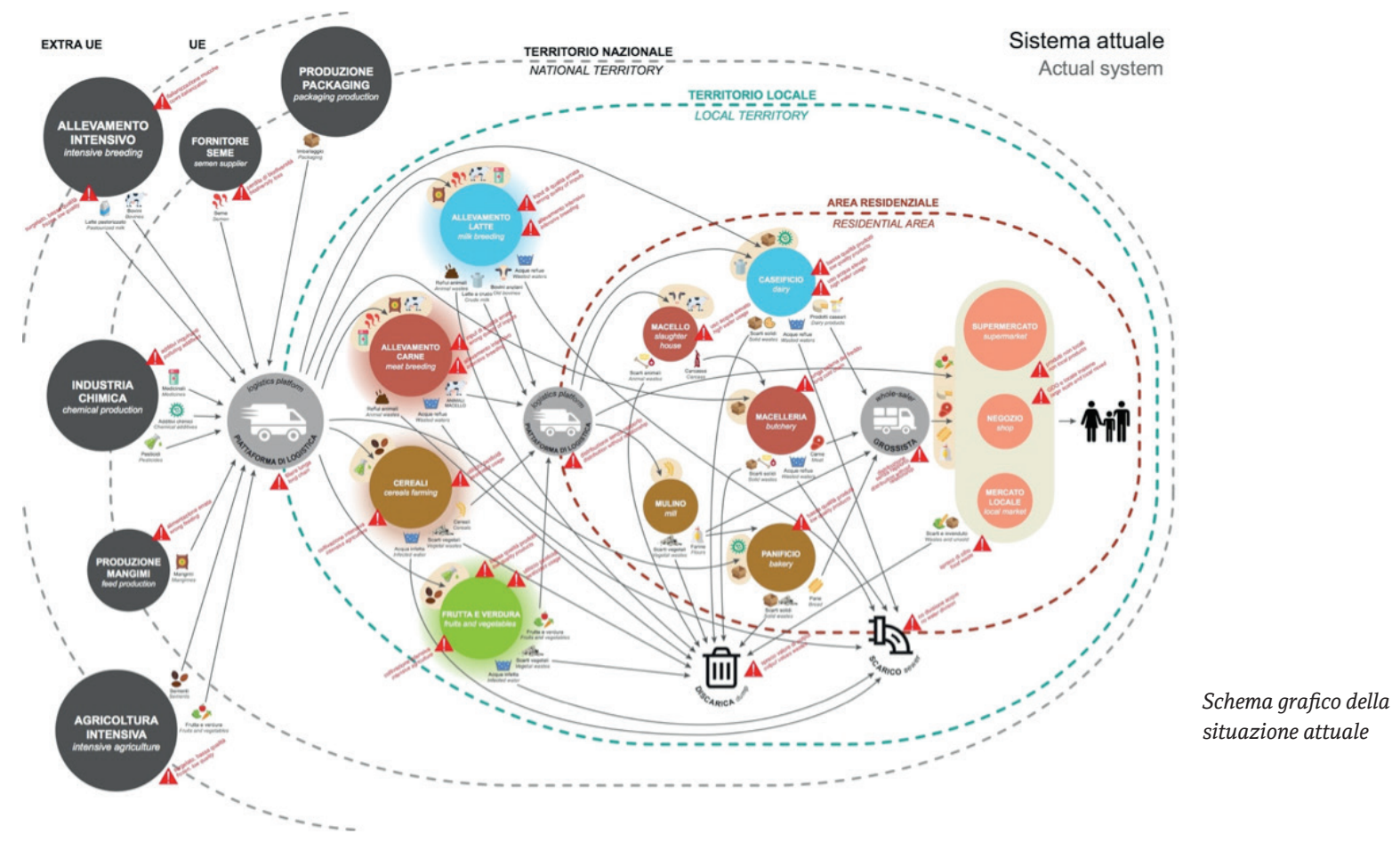

I prodotti diventano così globali ed il consumatore tende ad acquistarli, per la grande quantità di merci sempre disponibili, dalle Grandi Distribuzioni Organizzate (GDO) che si riforniscono dove il prezzo risulta minore. Si generano di conseguenza catene di distribuzione, del freddo e di mediatori che, attraverso piattaforme di logistica e trasporti su areo, nave e gomma, fanno arrivare i prodotti ovunque nel mondo.

Da un lato questa immensa mole di cibo in movimento incide sugli allevamenti e sulle coltivazioni trasformandoli in intensivi. Gli animali vivono in stabulazione fissa, con poco spazio disponibile, con antibiotici e alimentazione con integratori chimici per rendere più veloce il loro sviluppo; le colture utilizzano fertilizzanti di sintesi, per accelerare la crescita e la dimensione dei prodotti, e pesticidi che rendono le coltivazioni immuni da insetti e batteri, ma inquinano il suolo e la falda acquifera con una totale perdita di biodiversità.

Dall'altro il cibo, essendo visto come merce, risponde a criteri estetico-quantitativi (colore, forma e peso) e non organolettici. Come tale viene scelto o scartato all'origine e in ambito domestico, data la sempre abbondante presenza sul mercato, i consumatori sono indotti ad una sua scarsa considerazione con la conseguenza di un forte spreco del cibo comprato (circa il 30-40\%).

I territori considerati hanno però anche altre ricadute. 


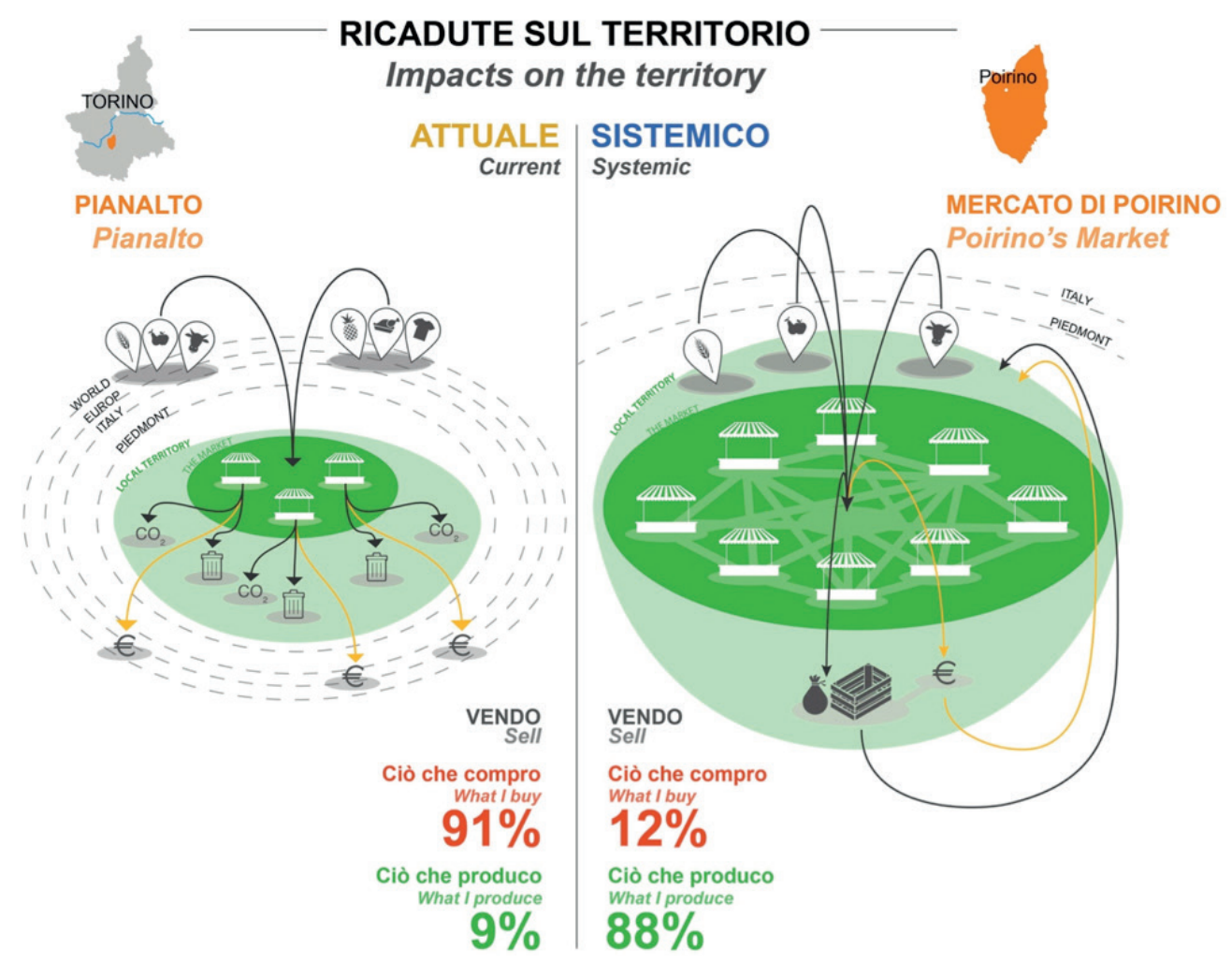

Comparazione del mercato di Poirino tra attuale e sistemico riferendosi alla presenza tipologica dei banchi di produttori (vendono ciò che producono) o di rivenditori (vendono ciò che comprano).

L'abitudine ormai ovvia di trovare in ogni stagione prodotti freschi che vengono da altri paesi e la sempre più invasiva presenza dei supermercati, favorita dalle amministrazioni locali per incassare nel breve ingenti oneri di urbanizzazione, ha trasformato anche i mercati locali e rionali. Questi da sempre sono stati il luogo dove i produttori locali hanno portato i propri prodotti freschi o trasformati, ma ora sono diventati anch'essi un centro commerciale all'aperto dove la produzione locale è diventata un'esigua minoranza.

L’indagine condotta su 3 mercati campione (Racconigi, Poirino e Piossasco) ha rivelato chei banchi di chi vende ciò che compra sono la stragrande maggioranza, mentre sono un'esigua minoranza quelli di chi vende ciò che produce.

Chi vende ciò che compra è interessato ad avere solo una differenza positiva per se tra quello che ha comprato all'ingrosso e quello che è riuscito a vendere. Il cibo è semplicemente denaro, il venditore asseconda le preferenze estetico-quantitative dei propri clienti e non è molto interessato a valorizzare le eventuali rimanenze di prodotti e di imballaggi. Tramite lui il denaro ritorna ai vari livelli multipli di provenienza delle risorse e non resta nel territorio locale che, come conseguenza, s’impoverisce nel tempo.

Chi invece vende ciò che produce è orgoglioso della propria produzione, che gli è costata lavoro e fatica, ne sa descrivere le qualità, da suggerimenti ai clienti e al termine del mercato riporta indietro sia gli imballaggi sia le rimanenze che può in parte trasformarle (in marmellate, succhi, sughi, etc) e in parte usarle come cibo per i propri animali. La sua presenza ed il suo lavoro contribuisco a valorizzare il territorio e la sua economia.

Perdendo queste piccole aziende agricole si perdono non solo la possibilità di avere prodotti freschi di stagione, ma si perde anche tutta la cultura materiale connessa alle coltivazioni (consociazioni di colture, rotazione di terreni, conservazione dei semi, etc.) e al rispetto dei beni comuni come acqua, canali irrigui, alberi e bosco.

Il bosco, con l'attuale cultura consumistica, è stato fortemente ridimensionato a favore dell'espansione agricola ed ha perso anche nel tempo la sua specifica caratteristica di essere di pianura (planiziale) o lungo un 
fiume (ripariale). Inoltre si è ridotto ad un'esigua presenza con poche varietà arboree utilizzate principalmente per il loro impiego industriale nella produzione di bancali, imballaggi, carta e la fauna selvatica non trovandovi protezione sconfina, come i cinghiali, nei terreni coltivati con tutte le conseguenze di rivolta dei proprietari.

In questo mero utilizzo strumentale e di consumo non viene neppure considerata la sua importantissima funzione di captare la $\mathrm{CO}^{2}$ con relativa generazione di ossigeno ed evaporazione di acqua!

\section{Un nuovo agire quotidiano}

Abbiamo visto, proprio all'inizio, quanto sia determinante e stravolgente il commisurare tutto partendo dalle quantificazioni economiche. Le azioni diventano utilitaristiche ed i rapporti tra le persone diventano molto superficiali e vuoti. La quantità ricercata fa tendere tutti ad una visione consumistica e strumentale azzerando la cultura, il territorio in cui si agisce, la valutazione delle conseguenze generate alle persone e all'ambiente. E' necessario un riscatto, una profonda percezione della realtà della nostra vita.

Bisogna far leva sulla consapevolezza delle persone in modo che ciascuno possa capire appieno le relazioni che le singole azioni quotidiane generano a ricaduta sugli altri e sull'ambiente in cui si vive; diffondere la riflessione che si fa parte di un insieme al quale tutti contribuiscono e dal quale tutti attingono.

Un riscatto, una presa di coscienza, una consapevolezza profonda di quanto sta accadendo per avviare azioni di forte ripensamento per ricostruire noi stessi, dall'interno, e per cominciare a ridefinire il paradigma culturale da mettere in atto.

Così enunciato il problema sembra essere molto facile da risolvere: basta seguire nuove linee guida per rimettere in sesto il tutto. Un po' come riassettare e pulire un ambiente in disordine; in un primo momento tutto si mantiene in ordine, ma con il passare del tempo lentamente ci si distrae e si ricade poco a poco nel disordine precedente.
Hanno semplicemente preso il sopravvento alcune azioni, che avevamo in precedenza, che hanno cancellato con la loro autoreferenzialità le relazioni positive di tutto l'insieme riportando quindi alla trasformazione negativa dell'intorno.

Oggi si vive immersi in un paradigma culturale di consumo che inghiotte e trascina ogni azione nella sua corrente vorticosa.

E' necessario acquisire una naturale conoscenza dei flussi di materia che generano il funzionamento dei vari processi, agricoli e produttivi, e delle relazioni che vengono attivate tra i diversi attori: si inizia in questo modo il cammino applicativo dell'Approccio Sistemico.

La valorizzazione di beni comuni, che possono offrire a tutta la comunità le proprie risorse, è la prima attenzione su cui focalizzare l'interesse. Tra questi il bosco costituisce una presenza fortemente significativa non solo per la collettività ma anche per le attività agricole in generale in quanto contribuisce significativamente al mantenimento delle risorse idriche, alla purificazione dell'aria, all'incremento dell'acqua e accoglie tutta una flora e una fauna selvatica che in esso trovano il loro habitat naturale. Inoltre la presenza in esso degli uccelli contrasta la diffusione degli insetti nocivi nelle coltivazioni mentre quella degli insetti utili, oltre all'apporto essenziale nell'impollinazione, collabora con gli uccelli alla salvaguardia dell'agricoltura. 

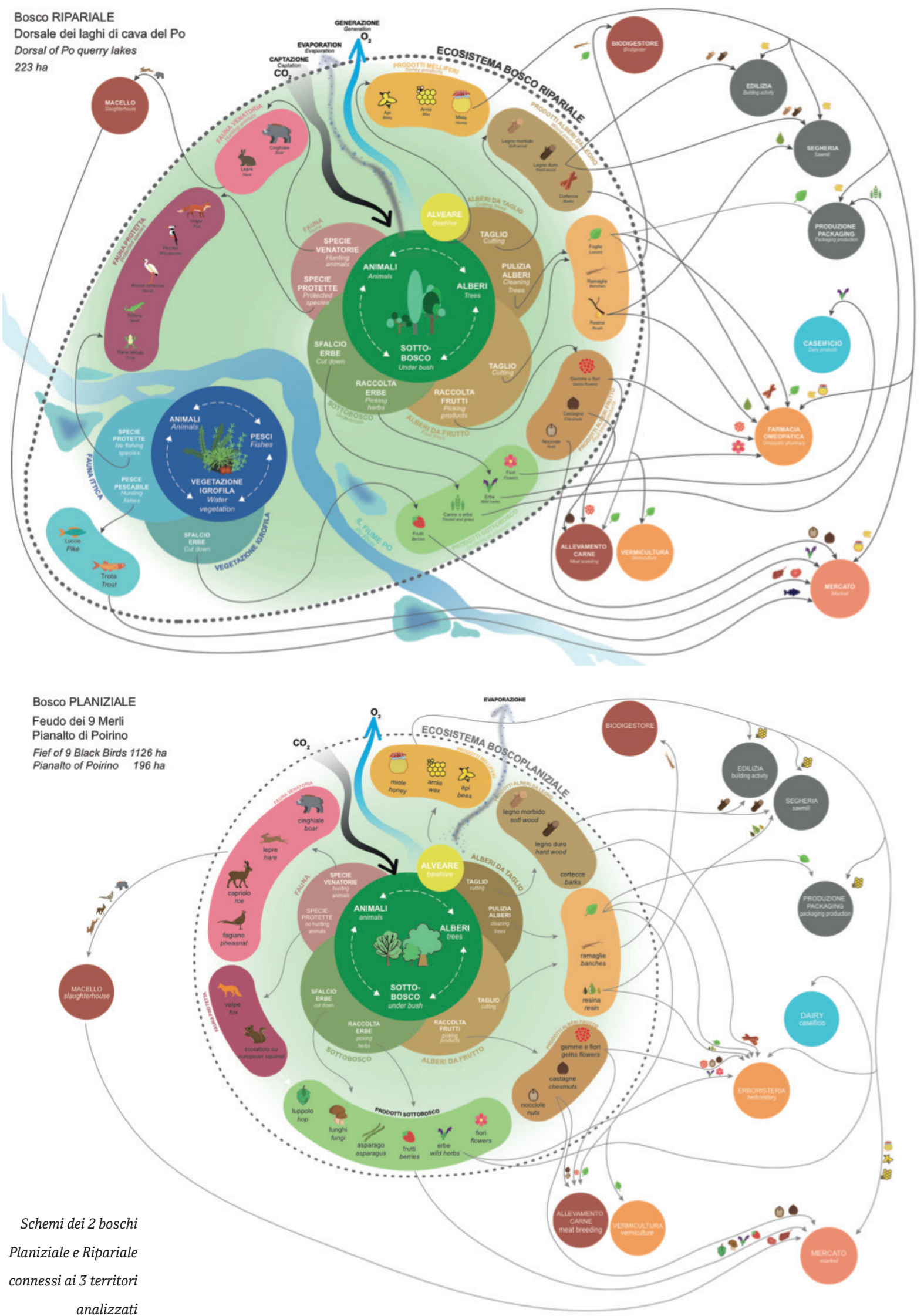
Per questi motivi ed anche per la grande presenza spontanea di risorse del sottobosco (dai funghi, ai piccoli frutti, alle erbe aromatiche), della selvaggina ed anche del legno, si è deciso d'inserire una superficie di bosco perenne di 1 ha (ripariale o planiziale a seconda della collocazione) nelle piccole aziende agricole (di 10-12 ha), dei territori di studio, come loro bene singolo, ma con ricadute generali per la comunità, in modo da rigenerare la ricchezza propria del territorio.

\section{Macro sistema territoriale}

Le singole attività primarie sono state sviluppate progettualmente per ricostruire le filiere locali (Latte, Carne, Cereali, Frutta \& Verdura e Bosco) in modo da poter approfondire tutti i nuovi rapporti relazionali ed i flussi di output-input che le collegano alle altre attività e inoltre per evitare di acquisire le risorse da territori lontani. Questo lavoro di approfondimento e di consolidamento delle diverse filiere evidenzia come le singole imprese siano legate le une alle altre, in un collegamento vicendevole generativo di flussi e relazioni, sino ad arrivare al cliente finale. L'ambito in cui sviluppare il sistema è il territorio locale: si vede come si crei un'unione inaspettata tra le realtà locali a partire dalle attività primarie via via a quelle generate prevedibili come pasticceria, bar, ristorante, macelleria, panificio o a quelle inaspettate come birrificio, malteria, erboristeria, edilizia, farmacia, laboratorio di fermentazione o a quelle di servizio generale come fitodepurazione, itticoltura, biodigestore, produzione di energia rinnovabile sino a raggiungere il mercato locale.

Inaspettatamente si constata come tutte le risorse (tranne per il sale e lo zucchero, anche se quest'ultimo potrebbe essere prodotto localmente dalle barbabietole o dalla frutta) siano reperibili sul territorio locale!

Quando poi si uniscono le diverse filiere ci si sbalordisce del risultato ottenuto perché nasce un macrosistema della nutrizione che rappresenta la possibile vita naturale e reale che potrebbe avere il territorio con le sue risorse. E' un fantastico corpo vivente in cui tutte le attività sono i singoli organi che si relazionano l'uno con l'altro e i flussi sono le sue arterie e vene. Ognuno è connesso utilmente con tutti gli altri.
L'aver spostato l'attenzione qualitativa non solo sui propri prodotti, ma anche sul processo produttivo, porta a generare output di qualità che sono input (risorsa) per altre attività. Chi li riceve li controlla qualitativamente prima di usarli e con questa azione, ripetuta continuamente all'interno del sistema, tutte le produzioni sono controllate ad ogni scambio. Tutto il sistema generato si autocontrolla ed offre ottimi prodotti.

Questo significa che la qualità non è rappresentata da una certificazione singola, ma semplicemente dalla propria appartenenza al sistema esistente.

Le stesse fattorie, che producono ciascuna in prev alenza o Latte o Carne o Cereali o Frutta e Verdura, sono state ridefinite considerando che le coltivazioni devono essere poste in consociazione tra di loro e anche con la prevista rotazione dei terreni. In questo modo durante tutto l'anno si possono avere diversi raccolti con un introito costante ed è anche visivamente interessante la trasformazione che contribuisco a dare al paesaggio nell'evolversi delle stagioni.

In questo nuovo clima collaborativo possono nascere anche delle relazioni tra aziende diverse (Latte con Frutta $\&$ Verdura e Cereali con Latte) in cui, per esempio, le mucche da latte in certe stagioni possono pascolare nei terreni delle produzioni di Frutta \& Verdura o Cereali in uno scambio positivo reciproco di concimazione e di pascolo che consolida la consapevolezza collettiva dell'agire.

Il sistema generale migliora la propria resilienza e ne è sempre più rafforzato.

Molti altri sono i benefici che la messa in pratica dell'Approccio Sistemico contribuisce a generare: dal rendere la struttura sociale molto coesa e collaborativa, al tutelare spontaneamente l'ambiente proprio con le produzioni delle varie attività, al diffondere a tutto il sistema sia benessere sia lavoro per tutti i residenti.

Ora si possono capire meglio le critiche mosse precedentemente al sistema lineare attuale e inoltre le varie attività, che sono i componenti del Macrosistema, approverebbero che qualcuno ritorni a coltivare con pesticidi oppure che si apra un supermercato? 


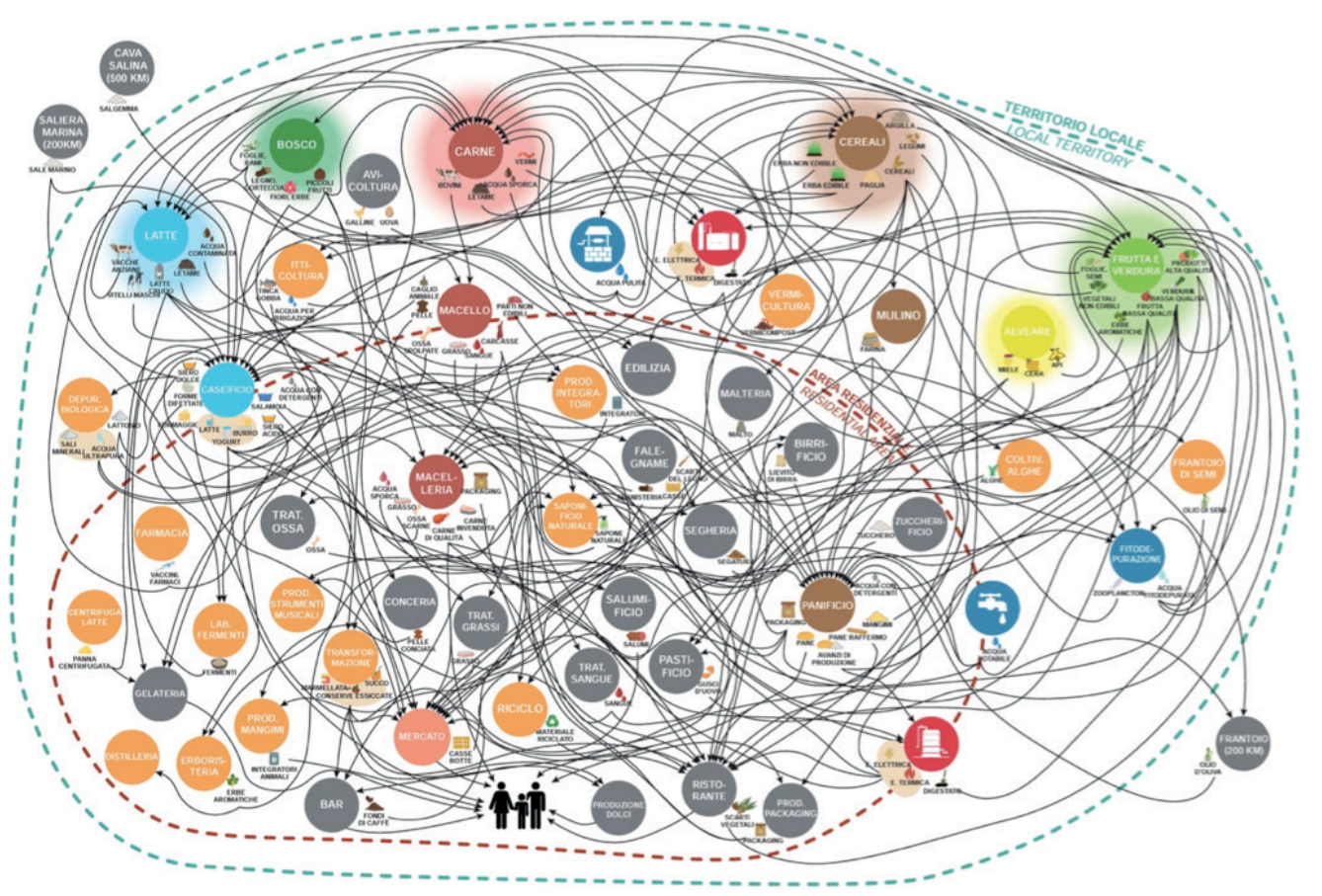

E’ fondamentale il passaggio di transizione al nuovo sistema.

Il passare dalla situazione lineare a quella sistemica non può certamente avvenire di colpo; non è come spegnere un interruttore e accenderne un altro, bisogna modificare gradualmente il modo corrente di agire e di pensare. E' necessario cominciare ad avviare delle relazioni, sperimentare, confrontarsi, condividere i risultati raggiunti, conquistare una consapevolezza non solo singola, ma collettiva.

Bisogna procedere a piccoli passi costruendo piccoli gruppi di relazione: un ristorante con i suoi fornitori, una pasticceria con un mulino ed una azienda di cereali e latte, una birreria con una malteria e con una coltivazione di orzo, e così via in modo che si mettano in azione volontariamente diverse esperienze che a poco a poco potranno avviare relazioni con altre attività e gradualmente giungere a realizzare il Macrosistema territoriale nel suo complesso.

E' crescere apprendendo. Questa evoluzione continua propria dell'Approccio Sistemico genera delle influenze positive anche agli altri diversi livelli del sociale, del culturale, dell'etico, dello scientifico, del produttivo e dell'economico.

\section{Evoluzione stagionale del mercato e del territorio}

Nella realtà sistemica il mercato locale riprende nuovamente vita tanto che nella proiezione effettuata dalla ricerca progettuale nei territori sulla composizione di 3 mercati campione (Racconigi, Poirino e Piossasco) risulta che i banchi di chi vende ciò che produce diventano la stragrande maggioranza, mentre si trasformano in un'esigua minoranza quelli di chi vende ciò che compra. 
Si passa dall'impoverimento all'arricchimento del territorio locale.

Il mercato, in questo nuovo vigore, può esprimere tutta la forza, la bellezza e la ricchezza del territorio che, nel trascorrere delle stagioni, è formata dalla continua variazione dei prodotti freschi e trasformati.

\section{SYSTEMIC MARKET Fief of 9 Blackbirds}

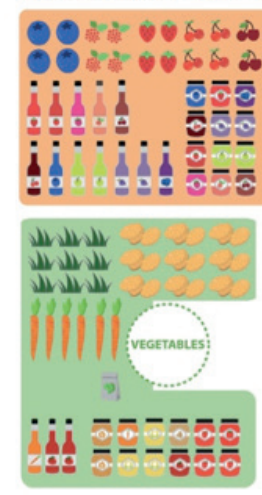

spring

SYSTEMIC MARKET Fief of 9 Blackbirds

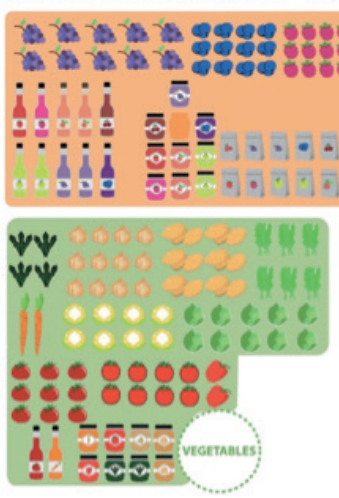

*summer

SYSTEMIC MARKET Fief of 9 Blackbirds

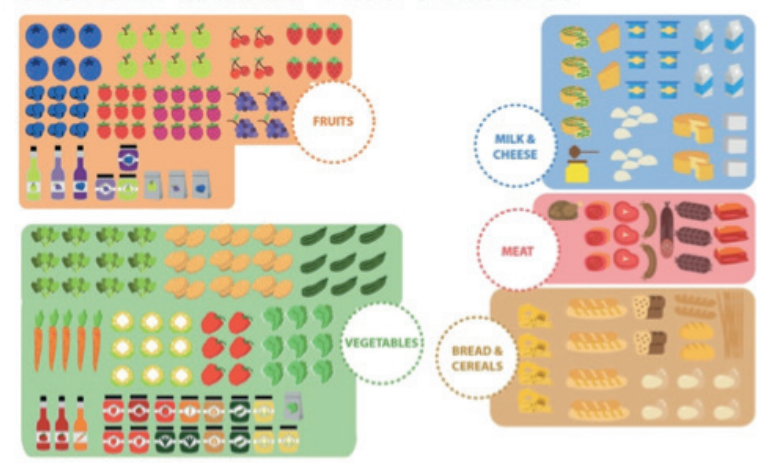

* autumn
SYSTEMIC MARKET Fief of 9 Blackbirds

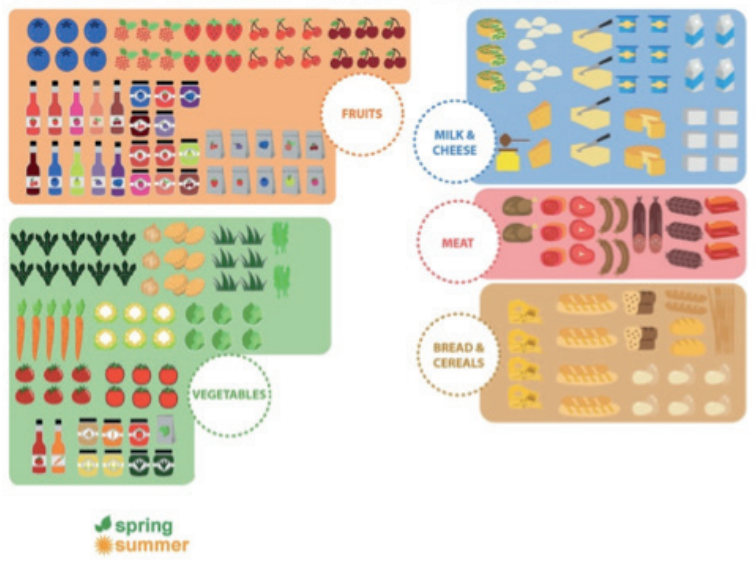

SYSTEMIC MARKET Fief of 9 Blackbirds

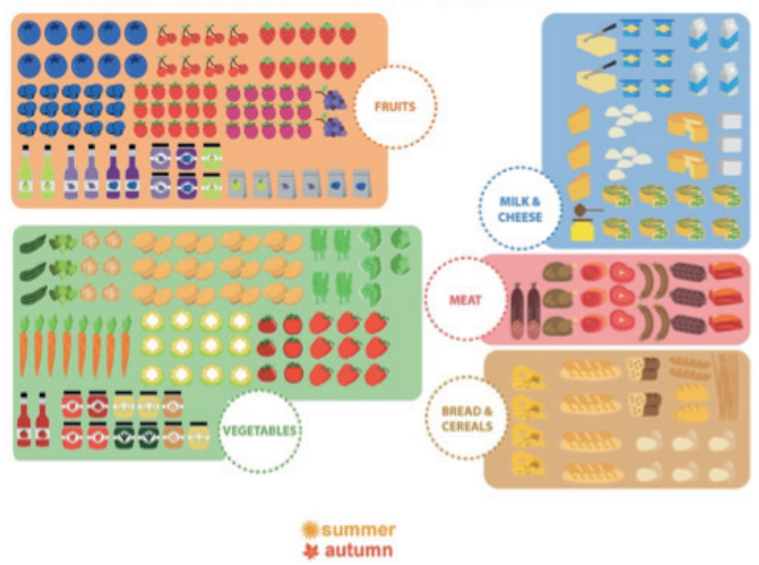

SYSTEMIC MARKET Fief of 9 Blackbirds

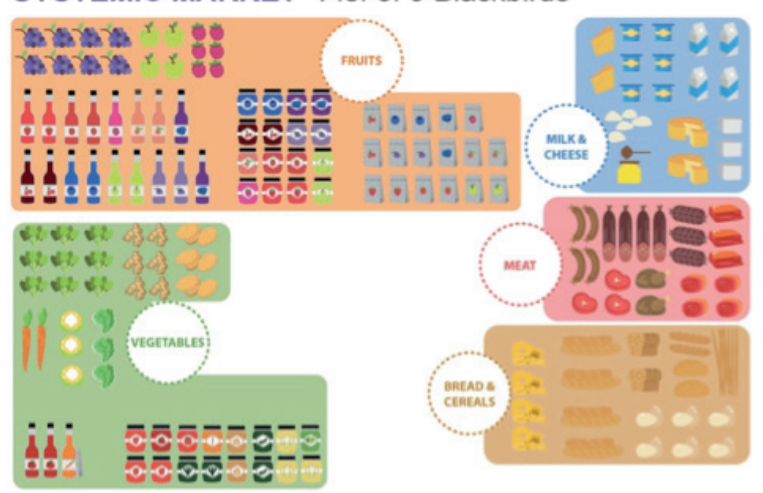

* autumn 
SYSTEMIC MARKET Fief of 9 Blackbirds
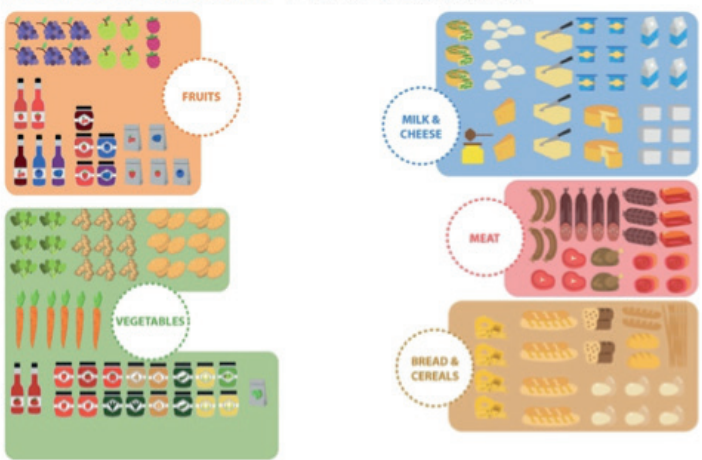

\% winter
SYSTEMIC MARKET Fief of 9 Blackbirds
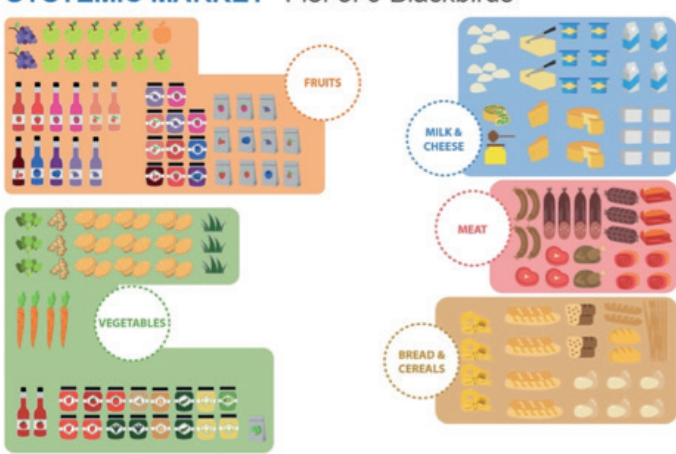

spring

Schemi dell'evoluzione del mercato del feudo dei 9 merli in relazione alle stagioni.

Il tempo è un valore fondamentale nella nostra vita. La scandisce nel suo trascorrere formando una lunga catena di avvenimenti e situazioni che sono la nostra storia personale. Noi siamo la somma di tutto questo, ma in ogni momento siamo quello che stiamo vivendo, abbiamo attività diverse a seconda del giorno o della notte, il nostro umore muta a seconda delle situazioni che ci coinvolgono, ...

Un territorio è anch'esso così, è la sommatoria annuale nel complesso dei suoi 365 giorni, ma ogni giorno di ogni stagione è diverso.

E' interessante poterlo vedere nella sua evoluzione stagionale, ne afferriamo la sua complessità. La visione stagionale ci offre risorse via via diverse che danno vita a piatti tipici, a convivialità diverse. Muta il ritmo dei giorni ed anche dei lavori che si svolgono. Ricorrono feste e manifestazioni che lo animano con la partecipazione dei suoi abitanti. Cambia la percezione delle architetture e dei monumenti che diventano più imponenti o più intimi. Evolvono le sensazioni che ci offrono le stagioni dalla nascita primaverile, al fulgore estivo, al declino autunnale sino al riposo invernale.
L’Approccio Sistemico fa riemergere una ricchezza di risorse, di comportamenti, di relazioni sociali che costituiscono un'esperienza coinvolgente per chi viene a visitare un luogo che non conosce e invoglia a ritornare per poter vivere nuove e diverse esperienze: non un turismo superficiale mordi e fuggi che visita i luoghi come per fare compere, ma un turismo esperienziale che partecipa al ritmo di vita del territorio, che è interessato a visitare le attività che lo animano ed entrare così nel suo spirito in un arricchente rapporto culturale. 
Lo schema allegato è la visualizzazione di come le peculiarità di un territorio si evolvono nelle diverse stagioni.

Contesto SISTEMICO ANNUALE - PIANALTO DI POIRINO ANNUAL SYSTEMIC CONTEXT - POIRINO'S UPLAND

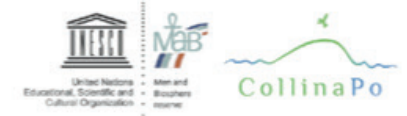

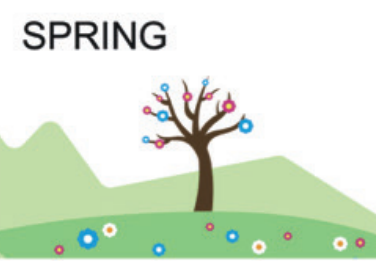

\section{SUMMER}

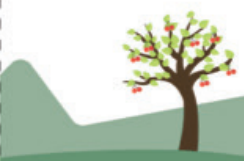

CULTURA

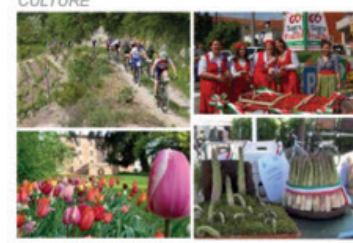

ARTIGIANATO

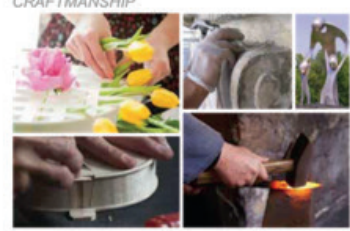

REALTA PRODUTTIVE

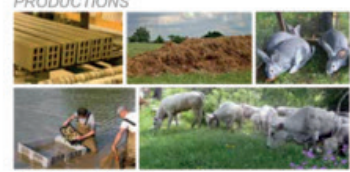

PIATTI TIPICI

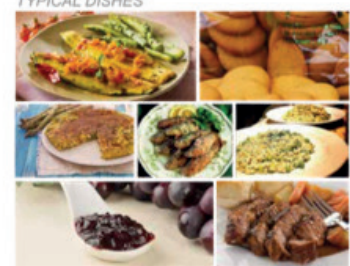

RISORSE

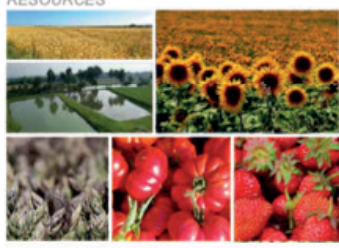

BIODIVERSITÀ

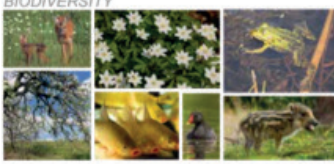

ARCHITETTURA

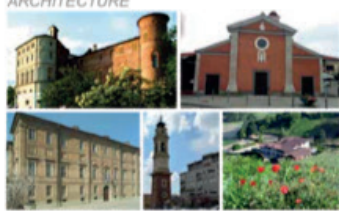

\section{CULTURA}

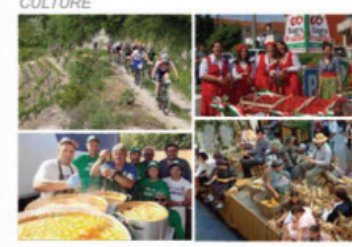

ARTIGIANATO

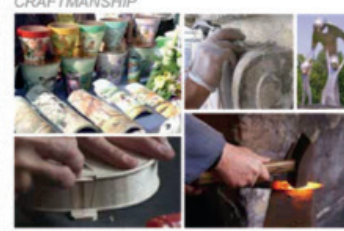

REALTÀ PRODUTTIVE

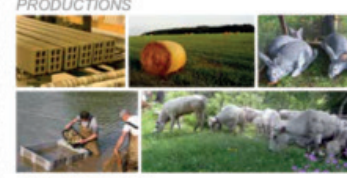

PIATTI TIPICI

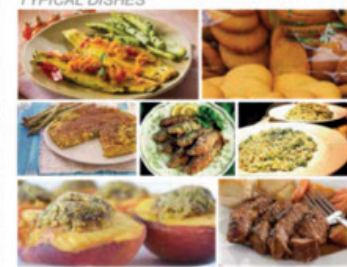

RISORSE

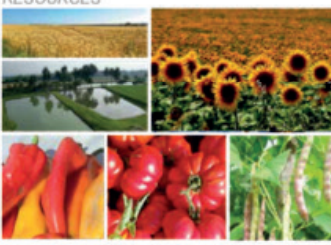

BIODIVERSITA

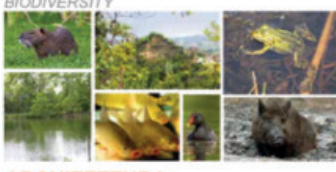

ARCHITETTURA
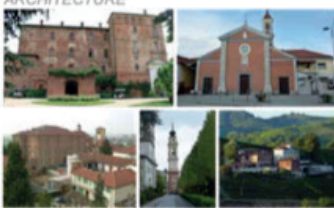

FALL

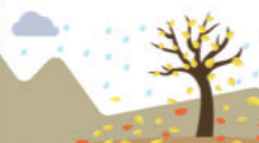

CULTURA

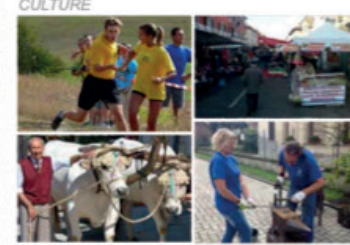

ARTIGIANATC
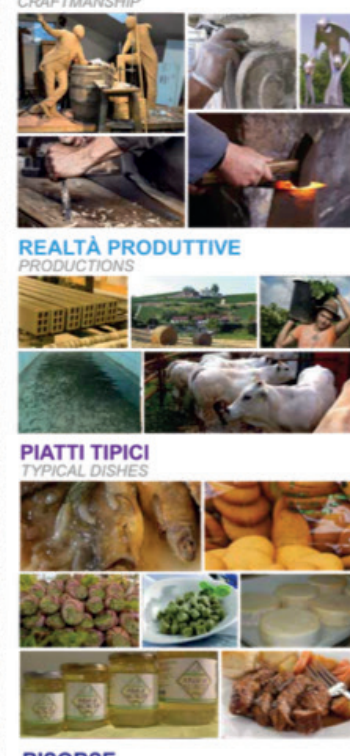

RISORSE

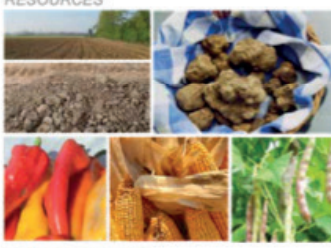

BIODIVERSITÀ

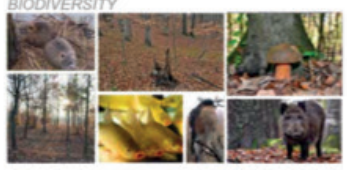

ARCHITETTURA

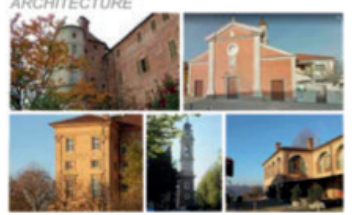

WINTER

CULTURA

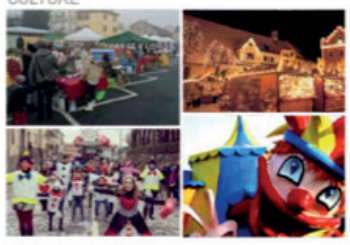

ARTIGIANATO

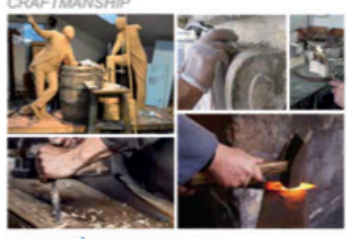

REALTÁ PRODUTTIVE

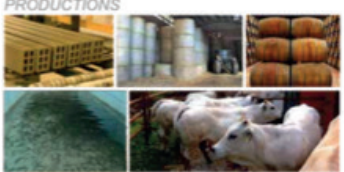

PIATTI TIPICI

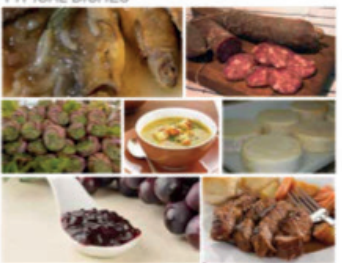

RISORSE

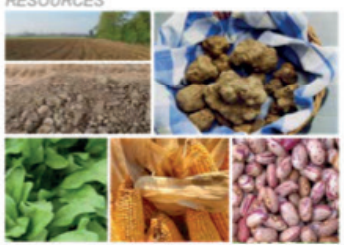
BIODIVERSITA

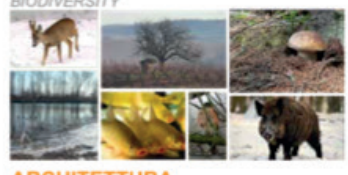

ARCHITETIURA

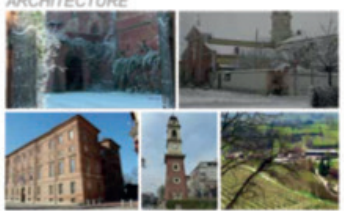




\section{Dieta alimentare e calcolo del fabbisogno alimentare corretto}

Avendo visto come si può ridefinire il sistema territoriale di produzione del cibo è ora importante approfondire come poter soddisfare le esigenze alimentari delle persone.

In generale quando si chiede a chi sono destinate le produzioni la risposta è univoca: il mercato.

Ma quale mercato? Purtroppo viene sempre sottintesa la sua reale specificazione "globale" e si entra quindi nella spirale della mercificazione del cibo.

L’Approccio Sistemico si occupa invece di quello "locale" e pone come determinanti, con semplice buon senso, questi prioritari ed essenziali fattori: le persone residenti e la loro salute.

Le abitudini alimentari attuali si basano su cibi prevalentemente fuori stagione, a lunga conservazione, assunti in maniera squilibrata e prodotti da catene industriali. Risultano in forte eccedenza le carni, gli insaccati, gli zuccheri (presenti in dolci vari, bibite, merendine), mentre sono molto in difetto la verdura e la frutta.
Questo regime alimentare ha purtroppo una stretta correlazione, unanimemente riscontrata da numerose ricerche mediche, di patologie che riguardano principalmente malattie del sistema cardio-circolatorio, il diabete, alcuni tipi di cancro ed altre metaboliche.

Per questo motivo il nostro Ministero della Salute, ma anche altri enti quali l'Organizzazione Mondiale della Sanità, preoccupati delle forti ricadute sulla salute delle persone hanno cercato di suggerire un regime alimentare bilanciato che viene denominato, per la forma geometrica generata, Piramide Alimentare.

L’Approccio Sistemico fa quindi riferimento alla Piramide Alimentare per definire il fabbisogno alimentare di cui si ha bisogno. Si ottiene il fabbisogno annuale richiesto per la popolazione del territorio con un semplice calcolo: la porzione degli alimenti suggeriti per persona si moltiplica per i giorni dell'anno e poi per il numero degli abitanti.

Questa è la vera quantità, necessaria e salutare, richiesta dal mercato locale!

\section{CONSEGUENZE DIETA ATTUALE}

\section{LINEARE}
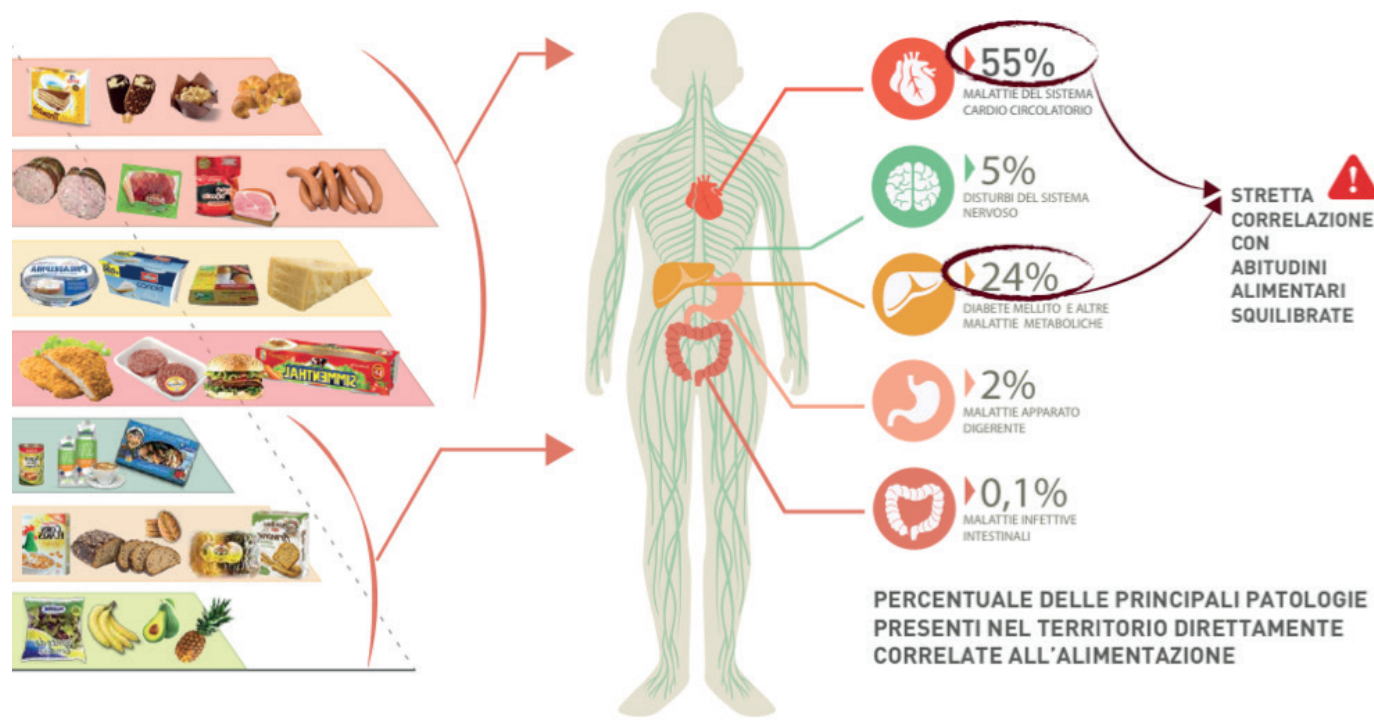

PERCENTUALE DELLE PRINCIPALI PATOLOGIE PRESENTI NEL TERRITORIO DIRETTAMENTE CORRELATE ALL'ALIMENTAZIONE

Schema della dieta attuale e ricadute sulla salute. 

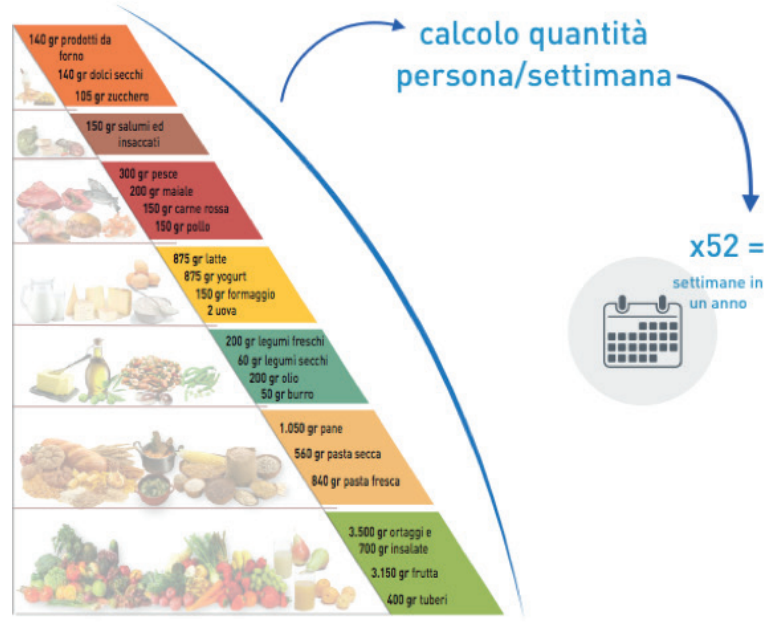

x 52 = quantità annua

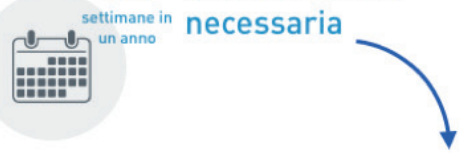

$\mathrm{X} \mathrm{n}^{\circ}$ abitanti $=$ FABBISOGNO ANNUALE

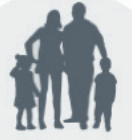

PER LA POPOLAZIONE

DEL TERRITORIO

\section{CONFRONTO DI VALORI}

società e alimentazione attuale e sociełà e alimentazione sistemica

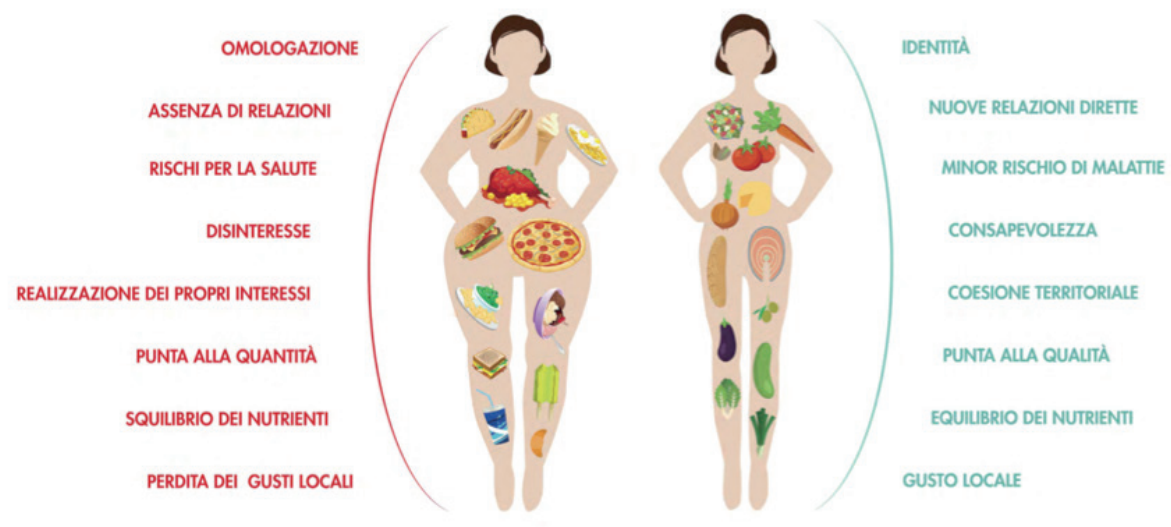

Visualizzazione del confronto tra i due regimi alimentari

\section{Riprogrammazione della SAU e collaborazione tra territori limitrofi}

Ogni comune nella propria pianificazione territoriale ha la suddivisione di come il proprio territorio sia utilizzato dalla superficie residenziale, a quella artigianale-industriale, a quella agricola e anche le loro consistenze dimensionali. Di quella agricola, denominata Superficie Agricola Utile (SAU), esiste anche l'utilizzo percentuale suddiviso per le varie coltivazioni. 
Prendendo quindi i dati dei comuni, di cui è composto il territorio analizzato, si ottiene la SAU totale con anche l'ammontare produttivo del territorio.

Dalla varie ricerche effettuate risulta che questo ammontare quasi sempre non raggiunge il soddisfacimento alimentare dei residenti perché le produzioni sono rivolte sempre al cosiddetto mercato globale che distribuisce, a livello nazionale e internazionale, le risorse seguendo solamente l'interesse finanziario. Il cibo, trasformato in bene monetario, è spostato e distribuito, con la super efficiente rete di logistica, nei luoghi che risultano economicamente più vantaggiosi a chi gestisce i loro flussi.

Si entra nella cosiddetta legge tanto cara agli economisti della domanda e dell'offerta.

Allora entriamo nel dettaglio di queste ultime.

In maniera molto semplice l'ammontare annuo della domanda è determinato, come abbiamo visto nel paragrafo precedente, moltiplicando il numero degli abitanti insediati per l'ammontare annuale di cibo per ogni persona, come suggerito dalla Piramide Alimentare, in modo da avere un'alimentazione corretta.

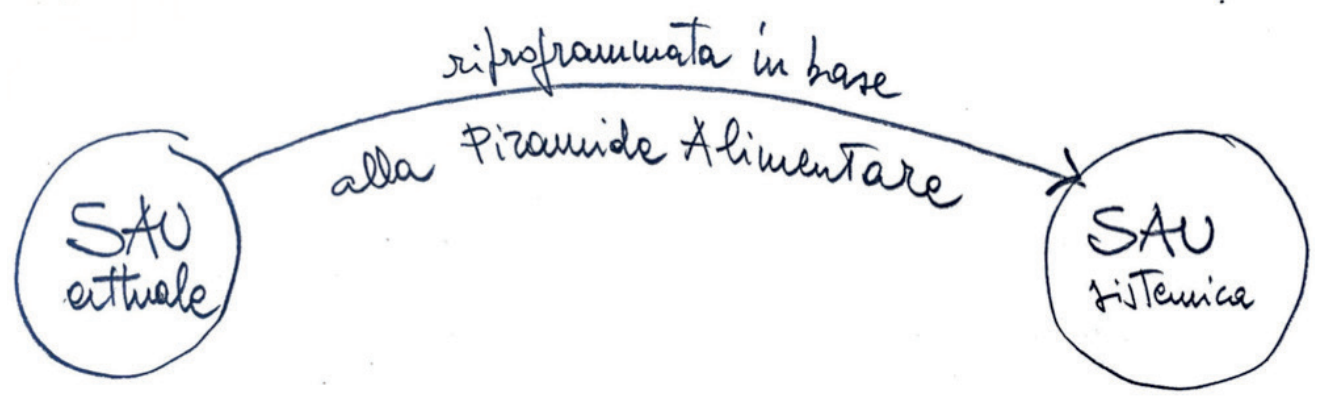

Schema della riprogrammazione della Superficie Agricola Utile (SAU).

La nuova offerta, nell'Approccio Sistemico, viene calcolata riprogrammando la SAU in base ai coefficienti produttivi per ottenere il riformulato fabbisogno alimentare annuo degli abitanti.

Sono due operazioni molto semplici e di buon senso che se applicate nelle diverse situazioni quasi dappertutto portano ad ottenere il 100\% ed anche oltre delle risorse necessarie con buona pace degli economisti. Il territorio può quindi offrire ai propri residenti quanto loro serve per vivere in salute.
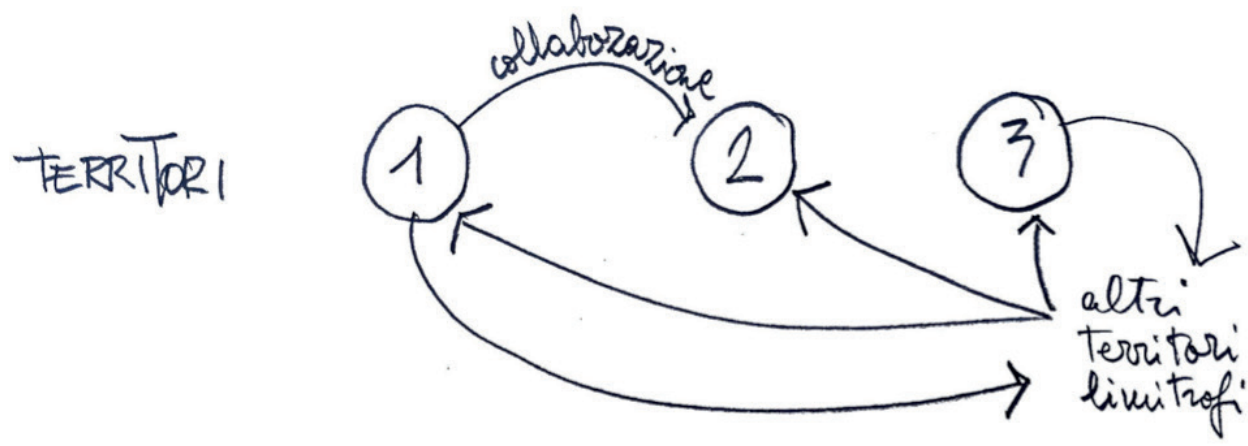
Nei casi in cui ci sia un'incidenza abitativa elevata e la SAU non sia sufficiente, come si verifica nel territorio dei Laghi di cava del Po, si dovrà guardare a quelli circonvicini per soddisfare le necessità delle risorse mancanti. Nel caso in questione l'adiacente Pianalto di Poirino ha infatti una disponibilità superiore al totale necessario per ogni abitante e può quindi offrire al territorio vicino quanto manca per il soddisfacimento del suo fabbisogno alimentare. Nasce quindi una predisposizione, propria di tutti i sistemi viventi, di operare vicendevolmente con il proprio intorno.
I Macrosistemi territoriali nel loro dialogare reciproco ottengono il risultato di rinforzare se stessi: i flussi d'interscambio con i vicini migliorano tutto il territorio interessato migliorando le loro relazioni sociali, culturali, produttive e rinforzando l'economia di ciascuno.

Gli schemi che seguono espongono in dettaglio i passaggi sinteticamente descritti.

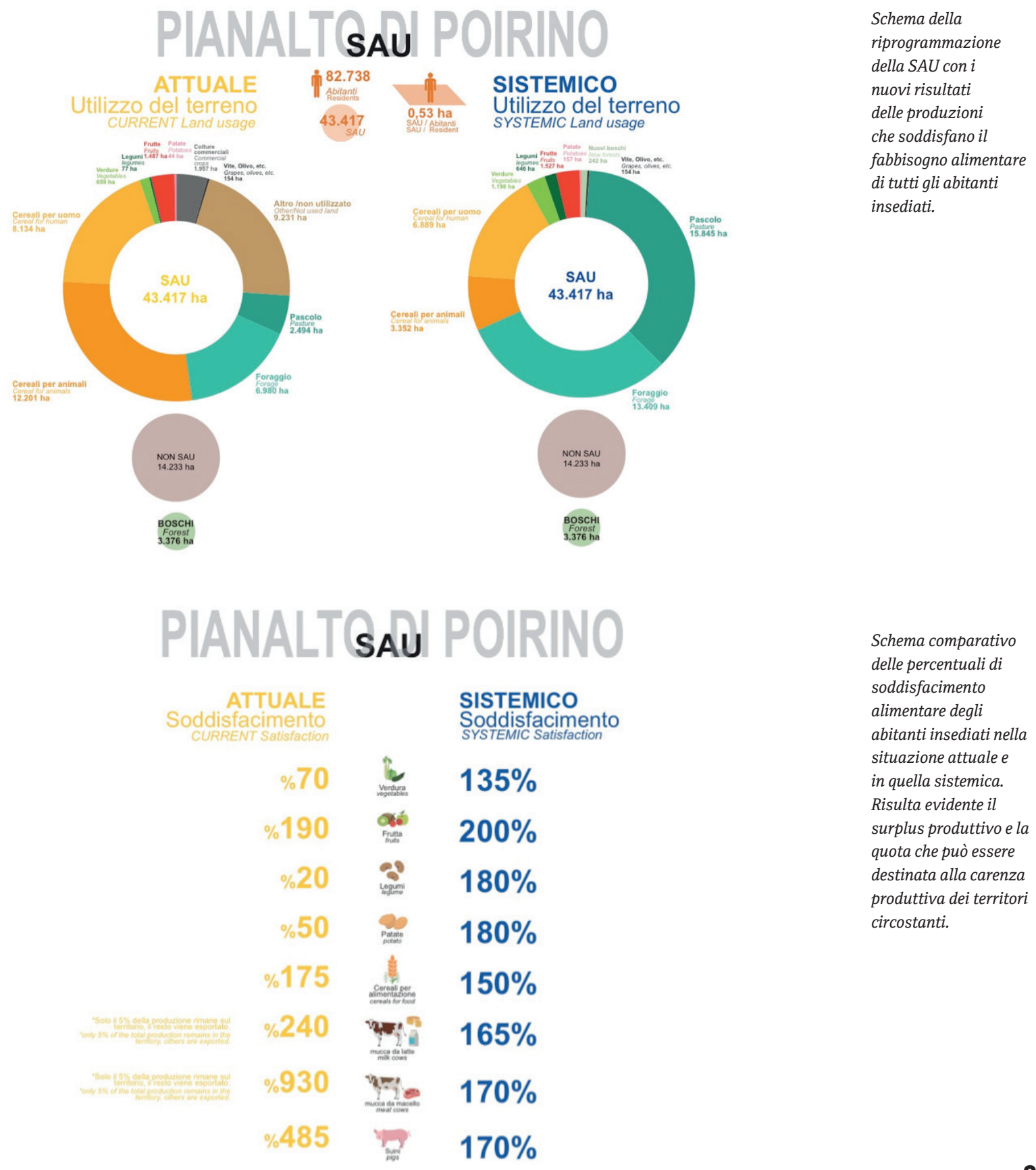




\section{PIANALTSAU POIRINO}

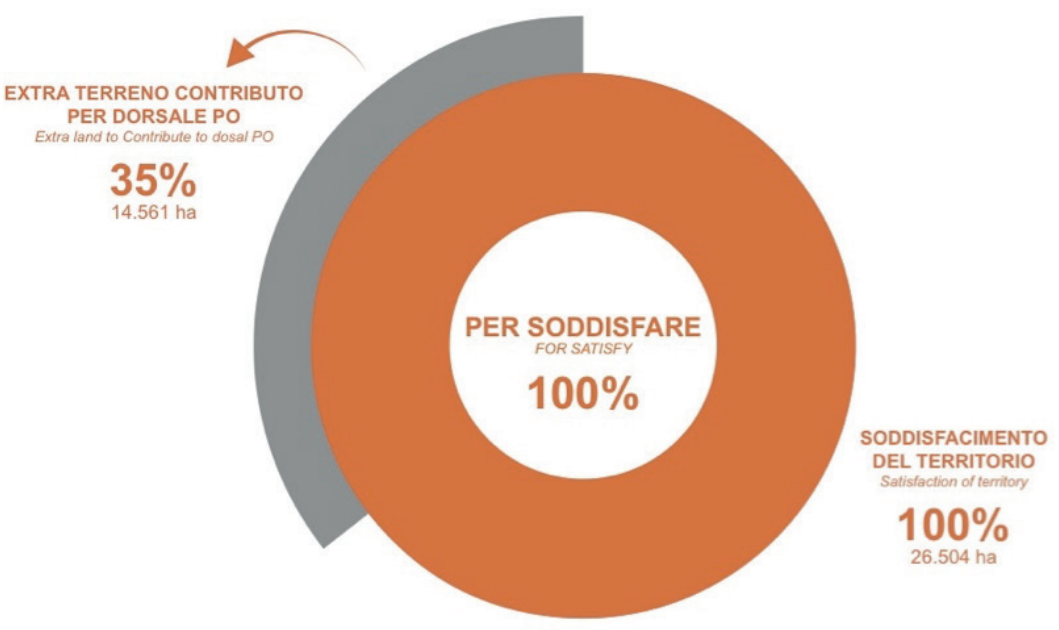

Schema grafico della SAU del Pianalto di Poirino con evidenziata la quota dedicata alla collaborazione con il territorio della Dorsale Laghi di cava del Po.

\section{Ricadute economiche nel territorio e benessere collettivo}

I risultati economici delle proiezioni dei singoli sistemi produttivi insediati nei diversi territori, ottenuti principalmente dalle micro e piccole aziende (MPI) ${ }^{5}$, sono strabilianti per le cifre indicate, ma saranno ancora più evidenti nella diffusione di benessere generale che apporteranno nelle diverse aree quando realmente il Macrosistema sarà operante.

L’applicazione del "Patto Partecipativo", in cui ogni attività partecipa con tutte le altre per giungere sul mercato locale (vedi nota a piè di pagina), ribalta i rapporti attuali conferendo ad ogni attore la possibilità di rimanere allo stesso tempo autonomo e fortemente relazionato al sistema che lui stesso contribuisce a formare assieme a tutti gli altri.

Il passaggio dal sistema lineare attuale a quello sistemico avviene gradualmente in un periodo di transizione molto attivo in cui si formano le filiere, si consolidano le relazioni tra le diverse attività, si riorganizzano le produzioni, si scambiano le esperienze dei risultati raggiunti tra le diverse esperienze e gradualmente l'insieme sociale assimila la consapevolezza del nuovo sistema.

E' un percorso apparentemente semplice nella sua definizione, ma complesso nell'acquisizione e metabolizzazione per i singoli come nuovo paradigma di scelta di vita.

5 vedasi a proposito di MPI e Patto Partecipativo, L. Bistagnino, “microMACRO” Edizioni Ambiente, Milano 2014. 


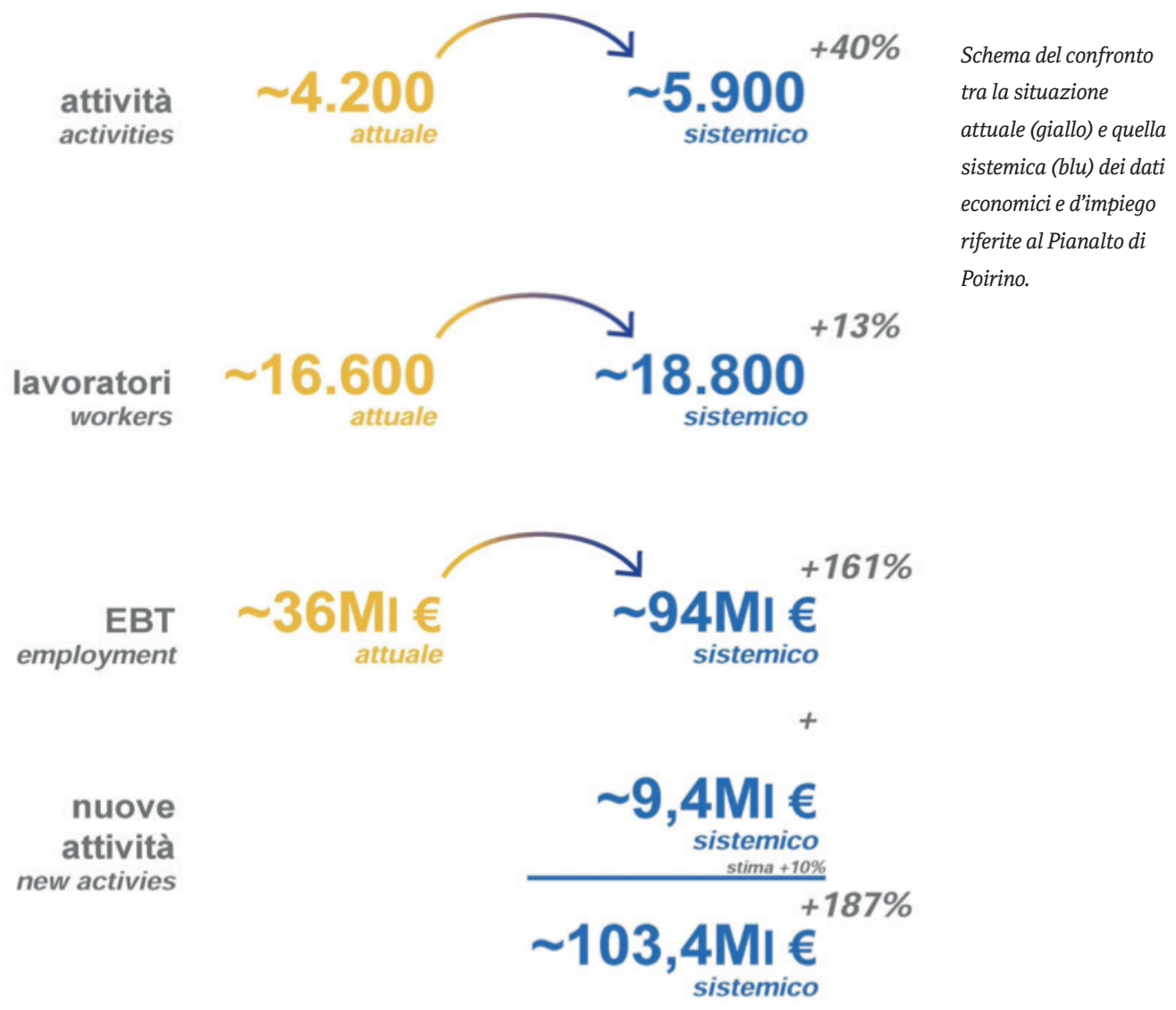

Le azioni che vengono svolte all'interno dei due diversi ambiti culturali dell'approccio lineare e di quello sistemico divergono completamente nelle loro finalità: nel primo è riferita egoisticamente a se stessi, nel secondo alla compartecipazione e alla comunità.

L'una mira al successo singolo, l'altra alla realizzazione di quello collettivo e in questa differenza si articolano i due diversi paradigmi culturali.

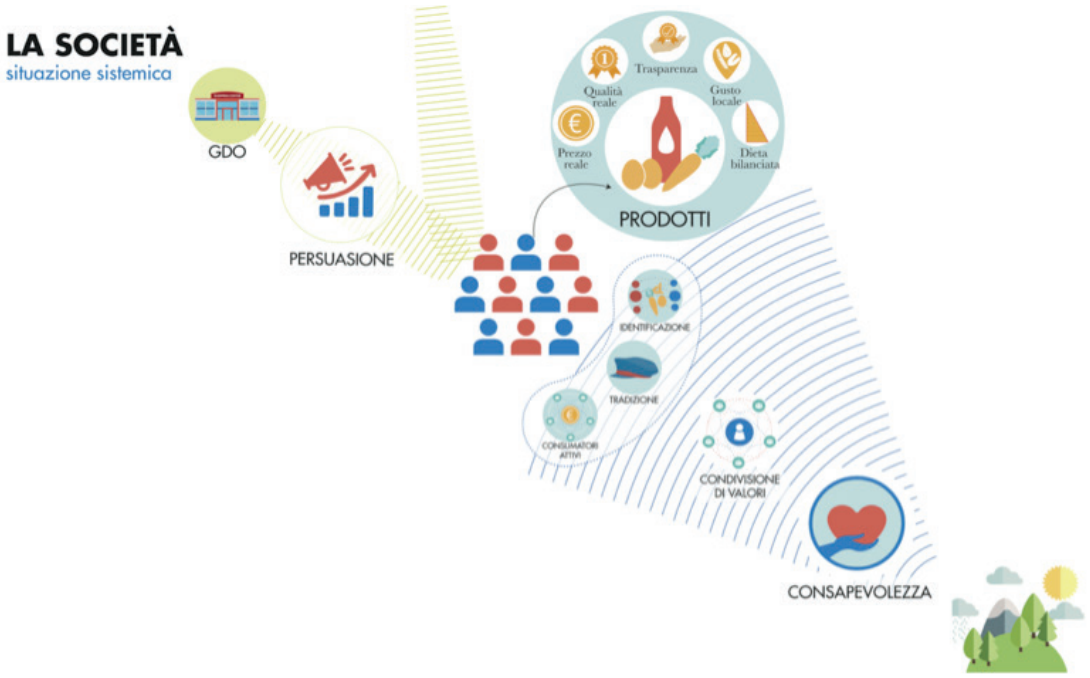




\section{LA SALUTE}
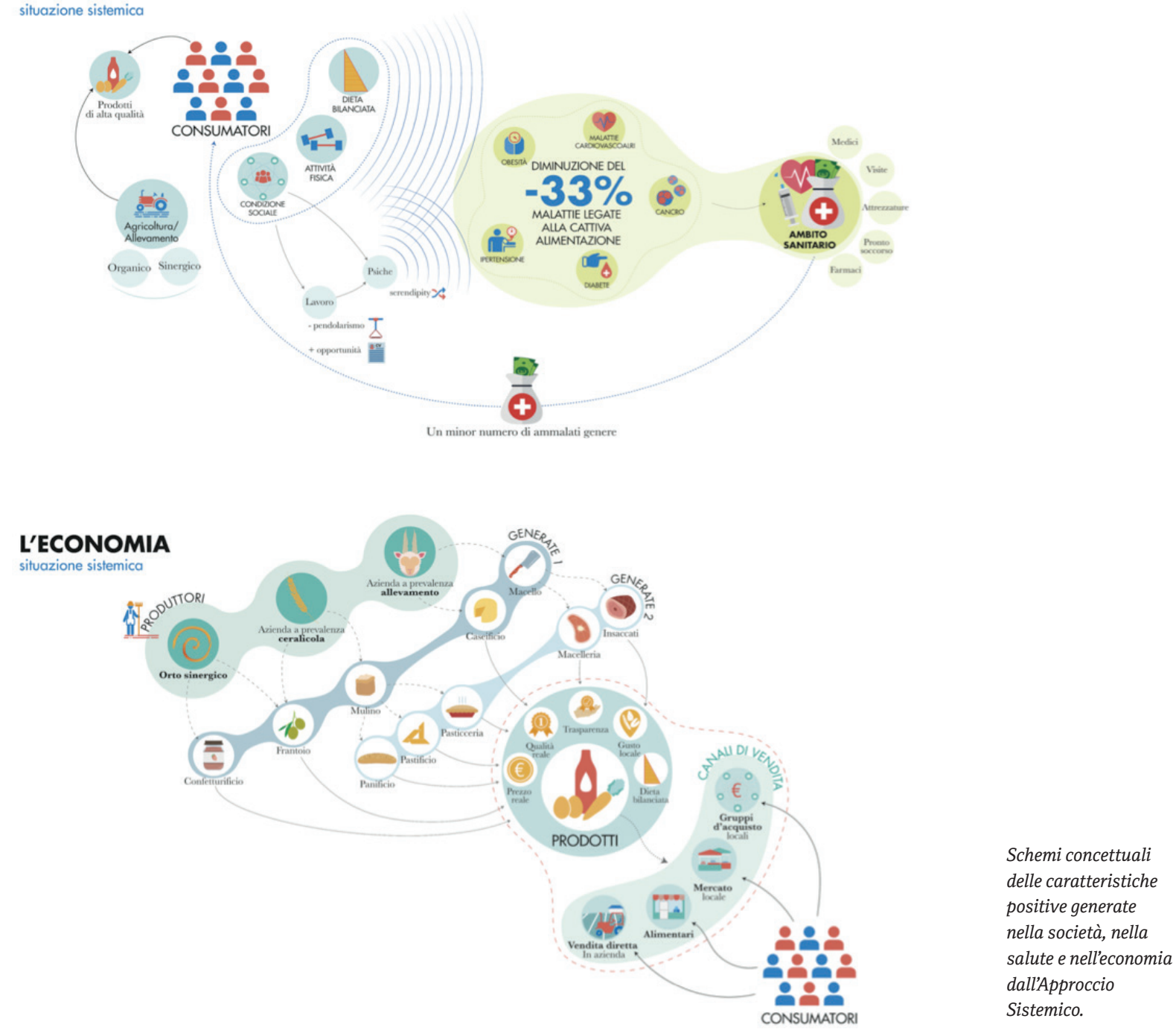

L'individualismo porta a costruire una struttura sociale gerarchica che si riconosce nella quantità di quanto si possiede, sull'accumulo del denaro e sulla competitività. Tutto ciò induce a creare un forte contrasto sociale e una prevaricazione economica che porta ad alleanze sovra territoriali tra i singoli, per aumentare la propria egemonia economica. Generano una rete globale di interessi monetari molto concentrati, completamente avulsi da ogni contesto e liberi da ogni condizionamento politico, territoriale e sociale. Chi opera in questo ambito compete con tutti gli altri per emergere e dominare il mercato sfruttando al massimo le risorse, rincorrendo il minor costo produttivo, anche delocalizzando l'attività, e operando in un contesto globale. Il denaro è il parametro di riferimento-valore che rende sottomessi tutti gli altri valori in quanto anch'essi devono misurarsi sulla quantità monetaria generata: l'operare, anche quello sociale, è impostato su quest'euforia collettiva di accumulo, di consumo, di quantificazione. 
Più volte si è fatto accenno alla necessità di riscatto, di riscossa profonda nel nostro agire quotidiano, di una consapevolezza che ci restituisca dignità. Questo legame unitario rende l'insieme compatto, flessibile, molto resiliente, la finalità di ciascuno è quella di tutti gli altri e si rafforza ciò a cui tutti tendono: il bene comune.

\section{la società}

\section{la cultura}

\section{l'ambiente}

\section{la produzione}

\section{l'economia}

\section{sono la risultante del nostro agire quotidiano}

La consapevolezza, che la collaborazione tra persone e la condivisione delle risorse (output-input) è la base della qualità di ogni rapporto, diffonde un'attenzione alla cura di quanto ci circonda e porta a valorizzare ogni risorsa umana e materiale. Il risultato economico diventa così la risultante finale positiva di tutte le azioni precedenti: offre un benessere condiviso e non la fonte contaminante del tutto.

Se si intende porre rimedio alla situazione attuale è necessario e fondamentale far partire dal basso, con l'Approccio Sistemico, la ricostruzione del nuovo sistema, seguirne con attenzione le evoluzioni ed attivare un dialogo multidisciplinare per poter far nascere i nuovi strumenti culturali e materiali di questo paradigma culturale coerenti alla vita del nuovo insieme.

Luigi Bistagnino é arquiteto, docente e coordenador de Departamento de Arquitetura \& Design do Politecnico di Torino (POLITO), Itália. Fundador do grupo de pesquisa em Design Sistêmico no Istituto POLITO. Atualmente é presidente da Systemic Approach Foundation e coordenador de Comitês Científicos no POLITO e na ECAM Lyon, na França, onde também leciona. 